\title{
A SETTING FOR GLOBAL ANALYSIS ${ }^{1}$
}

BY JAMES EELLS, JR.

Introduction. The primary aim of this report is to present a broad outline for a coherent geometric theory of certain aspects of nonlinear functional analysis. Its setting requires the calculus in topological vector spaces, differential geometry of infinite dimensional manifolds, and the algebraic and differential topology of function spaces. For the most part the developments are of quite recent origin, and at present the theory is in a fluid state (its growth depending strongly on its concrete applications). The beginnings of the subject may be traced to the work of Fréchet, Gâteaux, and Volterra; we refer to the text [73] of P. Lévy for an exposition of some early applications (especially in the calculus of variations and integrable differential systems) - and ask pardon for not presenting any historical perspective in the present survey.

About ten years ago it was formally recognized [29] that many of the function spaces which arise in global geometric mathematics possess a natural infinite dimensional differentiable manifold structure. That was not a great surprise; for

(1) Many of the most interesting manifolds of differential geometry are well known to have representations as function spaces of rigid maps. (E.g., Riemannian manifolds arise as the configuration spaces of dynamical systems, their cotangent bundles are interpreted as phase spaces, and their Riemannian metrics in terms of kinetic energy.)

(2) Much of the language of the classical treatment of the calculus of variations - and the penetrating viewpoint and methods of $M$. Morse-is that of a function space differential geometry. (E.g., the Euler-Lagrange operator of a variational problem has an interpretation as a gradient vector field, whose trajectories are lines of steepest descent.)

(3) Certain eigenvalue problems in integral and differential equations have interpretations in terms of Lagrange's method of multipliers, involving differential geometric ideas in infinite dimensions (e.g., focal point theory, and geometric consequences of the inverse

Based on an address delivered to the New York meeting of the Society on April 12,1965 , by invitation of the Committee to Select Hour Speakers for Eastern Sectional Meetings; received by the editors February 28, 1966.

1 Research partially supported by the National Science Foundation. 
function theorem). Throughout our exposition these examples have served as our guides.

Placing such classical problems in an appropriate geometric setting usually involves certain technical difficulties. For instance, choice of a suitable linear topology on the tangent spaces of the function space; choice of the right topological tensor products; choice of the appropriate type of differentiability. In certain cases these difficulties have been resolved-and then infinite dimensional differential geometry has led to new methods and results in classical analysis, global Riemannian geometry in finite dimensions, and algebraic topology. We have included here certain of these applications, the global calculus of variations being the most thoroughly developed of these (see $\S \S 8-10)$. Many others appear throughout the text as examples.

Comments and corrections for a preliminary draft of this article have been made by R. Abraham, R. Bonic, K. Jänich, J. McAlpin. I herewith express hearty thanks to them-along with my appreciation of the spirit of generosity and cooperation of the mathematical community, which in particular has made available to me in preprint or oral form a substantial part of the recent bibliographical references.

1. Linear space background. This section contains a review (with some new results) of certain aspects of linear space theory; in particular, we establish conventions and notation. For suitable expositions we refer to [13], [26].

(A) Let $E$ denote a locally convex topological vector space over the real number field $R$ (unless otherwise specified); thus $E$ is a Hausdorff topological vector space having a fundamental system of neighborhoods of its origin consisting of convex sets. We say that $E$ is a Fréchet space if its uniform structure is complete and metrizable. If $E$ is complete and has its uniform structure given by a norm $\left(x \rightarrow|x|_{E}=|x|\right.$ ) (resp., by an inner product $\left.(x, y) \rightarrow\langle x, y\rangle_{E}\right)$, we call $E$ a Banach space (resp., a Hilbert space); we use that terminology even though we may not have associated a distinguished norm for $E$ (in the family of equivalent norms determining the structure of $E$ ). It has been established very recently, through combined efforts of M. I. Kadec, C. Bessaga, A. Pelczyński, and R. D. Anderson, that all separable infinite dimensional Fréchet spaces are homeomorphic.

Say that a closed linear subspace $A$ of a Fréchet space $E$ is a direct summand if there is a closed linear subspace $C$ of $E$ which is supplementary to $A$; then $E$ is topologically isomorphic to the direct sum $A \oplus C$. Any finite dimensional subspace is a direct summand; so is any closed finite codimensional subspace (i.e., a subspace $A$ 
such that $\operatorname{dim}(E / A)<\infty)$, and any closed superspace of $A$ in $E$. Any closed subspace of a Hilbert space is a direct summand. Certain in teresting ideals in algebras of differentiable functions are direct summands; see [40, Chapter III 6; also $\$ 7 \mathrm{C}$ below]. On the other hand, Murray [84] has shown that $L^{p}(1<p \neq 2)$ always has closed linear subspaces without closed supplements.

(B) For homotopy theory the following fact is important (due independently to Palais [90], [92] and Svarc [115 in the case that $E$ is a Banach space]):

Let $E_{1} \subset E_{2} \subset \cdots$ be an increasing sequence of finite dimensional subspaces of a locally convex topological vector space $E$ whose union $U\left\{E_{k}: k \geqq 1\right\}$ is dense in $E$. For any subset $U$ of $E$ we define $U_{k}=U \cap E_{k}$ and $U_{\infty}=\operatorname{inj} \lim U_{k}$, the direct limit space of the sequence $\left(U_{k}\right)_{k \geq 1}$. If $U$ is open and paracompact, then the naturally induced map $U_{\infty} \rightarrow U$ is a homotopy equivalence.

In the same order of ideas [92]:

Let $E$ and $F$ be locally convex topological vector spaces, and $\phi: E \rightarrow F$ a continuous linear injection of $E$ onto a dense linear subspace of $F$. If $V$ is open in $F, U=\phi^{-1}(V)$, and both $U$ and $V$ are paracompact, then $\phi: U \rightarrow V$ is a homotopy equivalence.

(C) Given two locally convex topological vector spaces $E$ and $F$, we let $L(E, F)$ denote the vector space of all continuous linear maps $u: E \rightarrow F$. There are many useful (Hausdorff) locally convex topologies which can be put on $L(E, F)$-in particular, the following extremes: the weak topology (resp., the strong topology) is that of uniform convergence on the finite (resp., the bounded) subsets of $E$. If $E$ is a Fréchet space and $F$ is complete, then $L(E, F)$ is complete in the uniform structure of the strong topology. If $E$ and $F$ are Banach spaces with distinguished norms, then $L(E, F)$ is a Banach space with norm $\|u\|=\sup \left\{|u(x)|_{F} /|x|_{E}: 0 \neq x \in E\right\}$. For Banach spaces $E, F, G$ the canonical map $L(E, F) \times L(F, G) \rightarrow L(E, G)$, defined through composition of maps, is continuous.

Let $u \in L(E, F)$ and $F_{0}$ be a closed linear subspace of $F$. We say that $u$ is transversal to $F_{0}$ if the composition $E \rightarrow F \rightarrow F / F_{0}$ of $u$ with the coset map $\pi$ of $F_{0}$ is surjective and has kernel $E_{0}$ which is a direct summand of $E$. If $\pi \circ u: E \rightarrow F / F_{0}$ is surjective and $F_{0}$ has finite codimension in $F$, then $\operatorname{codim}\left(E, E_{0}\right)=\operatorname{codim}\left(F, F_{0}\right)$ and $u$ is transversal to $F_{0}$. If $E$ and $F$ are Banach spaces, then the subset of $L(E, F)$ consisting of those injections whose images are direct sum- 
mands is open in the norm topology on $L(E, F)$. So is the subset of those surjections whose kernels are direct summands; so is the subset of topological isomorphisms [2, p. 40].

(D) We will need to consider topological vector spaces of multilinear maps $E_{1} \times \cdots \times E_{k} \rightarrow F$ of locally convex topological vector spaces, which are jointly or separately continuous. In general that requires the theory of topological tensor products, a survey of which is given in [22]. For Fréchet spaces the separately continuous multilinear maps coincide with the jointly continuous ones. For Banach spaces $E, F$ let $(k \geqq 1) L^{k}(E, F)$ denote the Banach space of $k$-linear continuous (equivalently: bounded) maps $\alpha: E \times \cdots \times E \rightarrow F$; thus with distinguished norms on $E$ and $F$

$$
\left|\alpha\left(x_{1}, \cdots, x_{k}\right)\right|_{F} \leqq \text { const. }\left|x_{1}\right|_{E} \cdots\left|x_{k}\right|_{E} \text { for all } x_{i} \in E,
$$

and these norms induce a norm (whose topology is that determined by the projective tensor product) on $L^{k}(E, F)$. There is a canonical isometric isomorphism identifying $L^{k}(E, F)$ with $L\left(E, L^{k-1}(E, F)\right.$ ), where we agree to let $L^{0}(E, F)=F$. We denote by $S L^{k}(E, F)$ the closed linear subspace of $L^{k}(E, F)$ consisting of those $k$-linear maps which are symmetric in their arguments; similarly, $A L^{k}(E, F)$ denotes the subspace of alternating $k$-linear maps.

(E) Set $E^{*}=L(E, R)$, with strong topology. If $E$ is a Fréchet space, then $E^{*}$ is complete; $E^{*}$ is metrizable when and only when $E$ is a Banach space. If $u \in L(E, F)$, we let $u^{*} \in L\left(F^{*}, E^{*}\right)$ denote the adjoint of $u$, characterized by $\left\langle u^{*}\left(y^{*}\right), x\right\rangle=\left\langle y^{*}, u(x)\right\rangle$ for all $x \in E$, $y^{*} \in F^{*}$; here the left bracket denotes the canonical bilinear pairing $E^{*} \times E \rightarrow R$. Let $\operatorname{Ker}(u)=\{x \in E: u(x)=0\}$, and $\operatorname{Im}(u)=u(E)$; in case $u(E)$ is a closed subspace of $F$ we define the cokernel of $u$ by Coker $(u)=F / u(E)$.

We will be interested in certain subsets of $L(E, F)$, especially in case $E$ and $F$ are Banach spaces:

(1) Those $u \in L(E, F)$ with $u(E)$ closed in $F$; these maps are characterized (in case $E$ and $F$ are Fréchet spaces) as the maps $u$ carrying open sets of $E$ to open sets of $u(E)$. A map has closed range if it carries closed bounded subsets of $E$ in to closed bounded subsets of $F$. If $u$ has closed range, then

$$
\begin{array}{cl}
\operatorname{Im}(u)=\left\{y \in F:\left\langle y^{*}, y\right\rangle=0\right. & \text { for all } \left.y^{*} \in \operatorname{Ker}\left(u^{*}\right)\right\}, \\
\operatorname{Ker}(u)=\left\{x \in E:\left\langle x^{*}, x\right\rangle=0\right. & \text { for all } \left.x^{*} \in \operatorname{Im}\left(u^{*}\right)\right\} .
\end{array}
$$

(2) Those $u \in L(E, F)$ with closed ranges and finite dimensional kernels (resp., cokernels); we call these left-(resp., right-) Fredholm 
operators. If $E$ (resp., $F$ ) has finite dimension, then any $u \in L(E, F)$ is a left (resp., right) Fredholm operator. Those $u$ which are both left and right Fredholm operators are called Fredholm operators. For these their index is defined by ind $(u)=\operatorname{dim} \operatorname{Ker}(u)-\operatorname{dim}$ Coker $(u)$. If $u$ is Fredholm, then $\operatorname{dim} E<\infty$ if and only if $\operatorname{dim} F<\infty$. See [88] for general properties of Fredholm operators.

(3) An operator $k \in L(E, F)$ is compact if it maps bounded sets of $E$ into relatively compact sets of $F$ (i.e., sets whose closures in $F$ are compact); the compact linear operators form a closed subspace of $L(E, F)$, where $L(E, F)$ is given the norm topology. An operator $u \in L(E, F)$ is Fredholm if and only if there are maps $v_{1}, v_{2} \in L(F, E)$ such that $v_{1} u-I$ and $u v_{2}-I$ are compact (where $I$ denotes the identity map of the appropriate space). In particular, if $u$ is Fredholm and $k$ compact, then $u+k$ is Fredholm and ind $(u+k)=\operatorname{ind}(u)$. The totality of Fredholm operators forms an open subset of $L(E, F)$ with the index function constant on components; if $E$ and $F$ are Hilbert spaces, then two Fredholm operators with the same index are in the same component.

2. Smooth maps of Banach spaces. For the fundamental properties of the calculus in Banach spaces we refer to [23], [50], [64], [117], and to the older works [42]-[45], [73].

(A) Let $E$ and $F$ be real Banach spaces, and $U$ an open subset of $E$. There are many senses in which a map $\phi: U \rightarrow F$ can be considered as differentiable in $U$ (e.g., in the sense of Gâteaux, of Fréchet; pointwise or uniformly), the choice often depending on the applications under consideration. We proceed here as follows: A map $\phi: U \rightarrow F$ is (Fréchet) differentiable at $x_{0} \in U$ if there is a $\Phi \in L(E, F)$ such that

$$
\lim _{v \rightarrow 0} \frac{\left|\phi\left(x_{0}+v\right)-\phi\left(x_{0}\right)-\Phi(v)\right|_{F}}{|v|_{E}}=0
$$

for some (and hence any) choices of admissible norms on $E$ and $F$. Then $\Phi(v)$ is unique, and is henceforth denoted by $\phi_{*}\left(x_{0}\right) v$ or by $d \phi\left(x_{0} ; v\right)$, and called the differential of $\phi$ at $x_{0}$ in the direction $v \in E$. We have

$$
d \phi\left(x_{0}, v\right)=\lim _{h \rightarrow 0} \frac{\phi\left(x_{0}+h v\right)-\phi\left(x_{0}\right)}{h} ;
$$

conversely, if the right member exists as an element of $F$ in some neighborhood of $x_{0}$, is continuous at $x_{0}$, and $d \phi\left(x_{0}, v\right)$ is continuous in $v$ at $v=0$, then $\phi$ is Fréchet differentiable at $x_{0}$, and the right member 
defines its differential. If $\phi$ is differentiable at $x_{0}$, then $\phi$ is continuous there.

Suppose that $\phi$ is Fréchet differentiable at every $x \in U$; then we have the map $\phi_{*}: U \rightarrow L(E, F)$ defined by $x \rightarrow \phi_{*}(x)$, and we say that $\phi$ is of class $C^{1}$ if $\phi_{*}$ is continuous on $U$. If $\phi$ is Fréchet differentiable in $U$, then $\phi$ is $C^{1}$ if and only if $\phi_{*}$ is locally bounded and the limit (1) is locally uniform in $U$ (in the sense that for all $x_{0} \in U$ and $\epsilon>0$ there is a neighborhood $V$ of $x_{0}$ and $\delta>0$ such that $\mid \phi(x+v)-\phi(x)$ $-d \phi(x, v)|<\epsilon| v \mid$ for all $x \in V$ and $|v|<\delta)$. If $\phi_{*}=d \phi: U \rightarrow L(E, F)$ is Fréchet differentiable, then we can define $d^{2} \phi=d(d \phi): U$ $\rightarrow L(E, L(E, F))=L^{2}(E, F)$, which at each $x \in U$ is a symmetric continuous bilinear map of $E \times E \rightarrow F$; we say that $\phi$ is of class $C^{2}$ if $d^{2} \phi$ is continuous on $U$. Then define $\phi$ of class $C^{r}$ by induction, requiring that $d^{r} \phi: U \rightarrow S L^{r}(E, F)$ be continuous. Say henceforth that $\phi$ is differentiable on $U$ if it is $C^{r}$ for all $r \geqq 0$; let us agree that $d^{0} \phi=\phi$. The composition of $C^{r}$-maps is $C^{r}$; if $\phi$ is $C^{r}$ and $d^{r} \phi$ is $C^{s}$ then $\phi$ is $C^{r+s}$, and $d^{r+s} \phi=d^{s}\left(d^{r} \phi\right)$. Say that $\phi$ is analytic in $U$ if each point $x \in U$ has a neighborhood in which $\phi$ can be expressed by the absolutely convergent power series

$$
\phi(x+v)=\sum_{k=0}^{\infty} P_{\phi}^{k}(x, v) / k !
$$

where $P_{\phi}^{k}(x, v)=d^{k} \phi(x ; v, \cdots, v)$; again, the composition of analytic maps is analytic. If $E$ and $F$ are complex Banach spaces, we say that an analytic map $\phi$ is holomorphic; the elements of complex function theory are given in [24a], [50].

We have the following version of Taylor's formula:

Let $U$ be a convex neighborhood of $x_{0}$ in $E$ and $\phi: U \rightarrow F$ a $C^{r}$-map $(r \geqq 1)$. For any $1 \leqq j \leqq r$ there is a $C^{r-j_{-} m a p} R_{j}: U \rightarrow L^{j}(E, F)$ such that

where

$$
\phi\left(x_{0}+v\right)=\sum_{k=0}^{j-1} P_{\phi}^{k}\left(x_{0}, v\right) / k !+R_{j}\left(x_{0}+v\right)(v, \cdots, v),
$$

$$
R_{j}\left(x_{0}+v\right)(v, \cdots, v)=\int_{0}^{1} \frac{(1-t)^{j-1}}{(j-1) !} d^{j} \phi\left(x_{0}+t v\right)(v, \cdots, v) d t ;
$$

the integral is of course Banach space-valued. Furthermore, for every $\epsilon>0$ there is a $\delta>0$ such that for $|v| \leqq \delta$ we have

$$
\left|\phi\left(x_{0}+v\right)-\sum_{k=0}^{r} P_{\phi}^{k}\left(x_{0}, v\right) / k !\right| \leqq \epsilon|v| r .
$$

(B) If $\phi$ is a one-one $C^{r}$-map of an open set $U C E$ onto an open set $V \subset F$ and if its inverse $\phi^{-1}: V \rightarrow U$ is $C^{r}$, then we say that $\phi$ is a $C^{r}$ - 
diffeomorphism. Say that $\phi: U \rightarrow F$ is a (local) split $C^{r}$-imbedding if there is a representation $F=F_{1} \oplus F_{2}$, as the direct sum of Banach spaces with the following property: For each $x \in U$ there are neighborhoods $U_{x}, V_{x}$ of $x, \phi(x)$ and a $C^{r}$-diffeomorphism $\psi$ of $V_{x}$ onto an open subset of $F_{1} \oplus F_{2}$ carrying $\phi(x)$ on to 0 and such that $\psi \circ \phi$ is a $C^{r}$-diffeomorphism of $U_{x}$ onto an open subset of $F_{1} \oplus 0$. There is also the dual concept of (local) split $C^{r}$-projection.

The following inverse function theorem plays a fundamental role, both in the geometric and analytic aspects of the theory (see [23], [43], [64]):

Let $E$ and $F$ be Banach spaces, $U$ an open subset of $E, \phi: U \rightarrow F a$ $C^{r}$-map $(r \geqq 1)$. Then

(1) if $x \in U$ is a point for which $\phi_{*}(x): E \rightarrow F$ is injective and its image is a direct summand, then there is a neighborhood $U_{x}$ of $x$ in $U$ such that the restriction $\phi \mid U_{x} \rightarrow F$ is a split $C^{r}$-imbedding.

(2) If $\phi_{*}(x): E \rightarrow F$ is surjective and its kernel is a direct summand, then there is a neighborhood $U_{x}$ of $x$ such that $\phi \mid U_{x} \rightarrow F$ is a split $C^{r_{-}}$ projection.

(3) In particular, if $\phi_{*}(x)$ is bijective, then $\phi$ maps a neighborhood $U_{x}$ of $x C^{r}$-diffeomorphically onto a neighborhood of $\phi(x)$.

Example [108]. If $\phi: E \rightarrow F$ is a $C^{1}$-map with every differential $\phi_{*}(x)$ bijective and satisfying $\left\|\phi_{*}(x)^{-1}\right\| \leqq K$ for some $K \in R$ and all $x \in E$, then $\phi$ is a $C^{1}$-diffeomorphism of $E$ onto $F$. In particular, the conclusion is valid if $E=F$ is a Hilbert space, and $\left\langle\phi_{*}(x) v, v\right\rangle \geqq c|v|^{2}$ for some $c>0$ and all $x, v \in E$. That last condition is equivalent (for $C^{1_{-}}$ maps) to saying that $\phi$ is strongly monotone: $\langle\phi(x)-\phi(y), x-y\rangle$ $\geqq c|x-y|^{2}$ for all $x, y \in E$.

Closely related to the inverse function theorem are several implicit function theorems which have found varied analytic interpretations. See $[117, \S \S 17,26]$ for applications to integral equations, especially in the study of branch points of operators. The Nash-Moser implicit function theorem [83], [108] provides a very powerful iteration technique used to establish the existence of differentiable solutions of certain differential equations. Under certain conditions on the differential of $\phi$ a form of the inverse function theorem is valid even though $\phi_{*}$ may not be continuous [5].

(C) A basic problem [29] in the theory of infinite dimensional manifolds is that of showing the existence of sufficiently many nontrivial differentiable functions. Let us say that a Banach space $E$ is $C^{r}$-smooth if there is a nontrivial real valued $C^{r}$-function on $E$ with bounded support. (The support $S(\phi)$ of a function $\phi: E \rightarrow R$ is the closure of $\{x \in E: \phi(x) \neq 0\}$.) The following characterization is due 
to Bonic and Frampton (see [10], [11], [37] for this and for most of the examples below):

If $E$ is a separable Banach space, then the following properties are equivalent: (a) $E$ is $C^{r}$-smooth; (b) any open subset $U$ of $E$ admits $C^{r_{-}}$ partitions of unity, (i.e., for every open cover $\mathfrak{U}$ of $U$ there is a system $\left(\psi_{\alpha}\right)$ of nontrivial $C^{r}$-functions $\psi_{\alpha}: U \rightarrow R(\geqq 0)$ such that the set of all supports $S\left(\psi_{\alpha}\right)$ is locally finite (each $x \in U$ has a neighborhood which meets only finitely many of the $\left.S\left(\psi_{\alpha}\right)\right)$, each $S\left(\psi_{\alpha}\right)$ is contained in some element of $\mathfrak{u}$, and the sum $\left.\sum_{\alpha} \psi_{\alpha}=1\right)$; (c) for any continuous map $\phi$ of $U$ into a Banach space $F$ and any number $\epsilon>0$, there is a $C^{r}$-map $\psi: U \rightarrow F$ such that $|\phi(x)-\psi(x)|_{F}<\epsilon$ for all $x \in U$.

In a separable space $E$ satisfying these conditions it follows easily that any closed set $A$ is the precise locus of zeros of some $C^{r}$-function $\phi: E \rightarrow R$. Furthermore, if $A_{0}, A_{1}$ are disjoint nonvoid closed subsets of $E$, then there is a $C^{r}$-function $\phi: E \rightarrow R$ with $0 \leqq \phi(x) \leqq 1$ for all $x \in E, \phi(x)=0$ (resp., $\phi(x)=1$ ) when and only when $x \in A_{0}$ (resp., $x \in A_{1}$ ). The extent to which separability is needed in these results is not clear.

Any Banach space $E$ is $C^{0}$-smooth; in fact, we can use the metric structure of $E$ to construct locally Lipschitz functions which separate disjoint closed sets in $E$. Any separable Hilbert space is $C^{\infty}$-smooth, since the square of the norm is quadratic, and hence $C^{\infty}$. Restrepo [97] has shown that a Banach space $E$ has a $C^{1}$-norm (except at the origin) if and only if its conjugate space $E^{*}$ is separable; apparently little is known about spaces with $C^{r}$-norms $(r \geqq 2)$. Thus if $E^{*}$ is separable then $E$ is $C^{1}$-smooth. The Banach spaces $L^{p}$ of all Lebesgue $p$ th power $(1 \leqq p<\infty)$ summable functions $f:[0,1] \rightarrow R$ with norms

$$
|f|_{L^{p}}=\left[\int_{0}^{1}|f(t)|^{p} d t\right]^{1 / p}
$$

are $C^{\infty}$-smooth for $p$ an even integer $\geqq 2$; otherwise $(1 \leqq p<\infty) L^{p}$ is $C^{r}$-smooth if $r$ is the integer satisfying $r<p \leqq r+1$ (since its norm is $C^{r}$ except at the origin), but $L^{p}$ is not $C^{r+1}$-smooth. (This last assertion is implied by work of Kurzweil [63]; in particular, $l^{1}$ is not $C^{1}-$ smooth.) As an application, we observe that $L^{3}$ and $L^{4}$ are not $C^{3}$ diffeomorphic, although by a theorem of Mazur they are homeomorphic. The Banach space $c_{0}$ (the space of sequences of real numbers which converge to 0 , with supremum norm) is $C^{\infty}$-smooth; on the other hand, the space $C$ (all continuous functions $f:[0,1] \rightarrow R$ with supremum norm) is not $C^{1}$-smooth.

Certain maps which are not sufficiently differentiable are deter- 
mined by their boundary values [11]: Let $E$ be a Banach space containing a subspace isomorphic to $l^{1}$ (for example, take $E=L^{1}$ or $C$ or $C^{k}$ ), and $F$ a reflexive Banach space. If $\phi: \bar{U} \rightarrow F$ is a continuous map of the closure of a bounded connected open subset of $E$ which is $C^{1}$ on $U$, then $\phi$ (boundary $U$ ) is dense in $\phi(\bar{U})$.

(D) There are examples of $C^{\infty}$-functions $\phi: E \rightarrow R$ defined on Banach spaces $E$ such that the image in $R$ of the set of critical points (i.e., points $x \in E$ for which $\phi_{*}(x)=0$ ) is of strictly positive measure. Kupka [62] has constructed such a function for the case that $E$ is a separable Hilbert space. The following example is due to $\mathrm{R}$. Bonic.

ExAMPLE. Let $\alpha: R \rightarrow R(\geqq 0)$ be a $C^{\infty}$-function such that $\alpha(t)$ $=0(t \leqq 0), \alpha(t)=1(t \geqq 1)$. Let $E=L^{\infty}[0,1]$ and $\phi: E \rightarrow R$ be defined by $\phi(x)=\int_{0}^{1} \alpha(x(t)) d t$ for all $x \in E$. Then $\phi$ is $C^{\infty}$ on $E$; in particular,

$$
d \phi(x, v)=\int_{0}^{1} \alpha^{\prime}(x(t)) v(t) d t .
$$

For any $0 \leqq s \leqq 1$ let $x_{s}$ be the characteristic function of $[0, s]$. It follows that $\phi\left(x_{0}\right)=0, \phi\left(x_{1}\right)=1$; on the other hand, $\phi_{*}\left(x_{8}\right)=0(0 \leqq s \leqq 1)$. Thus $s \rightarrow x_{s}$ is a (discontinuous) path in $E$ on the image of which $\phi$ is not constant, and yet every point of which is a critical point of $\phi$.

Thus we cannot expect a complete generalization to infinite dimensions of the Brown-Morse-Sard theorem; however, (1) Sard [104] has recently obtained precise results on the Hausdorff measure of the image of the critical points of a $C^{r}$-map $\phi: U \rightarrow F$, where $U$ is an open subset of $\boldsymbol{R}^{n}$ and $F$ is any Banach space; (2) using the finite dimensional case and the inverse function theorem, Smale [111] has obtained the following version of that result:

Let $U$ be a connected open subset of a separable Banach space $E$ and $\phi: U \rightarrow F$ a $C^{r}$-map $(r \geqq 1)$. The critical set of $\phi$ is $C=\left\{x \in U: \phi_{*}(x)\right.$ is not surjective $\}$. Say that $\phi$ is a Fredholm map if for every $x \in U$ the differential $\phi_{*}(x): E \rightarrow F$ is a Fredholm operator; recall consequently that $F$ is infinite dimensional whenever $E$ is. The index of $\phi$ is the constant value ind $(\phi)=\operatorname{dimKer}\left(\phi_{*}(x)\right)-\operatorname{dim} \operatorname{Coker}\left(\phi_{*}(x)\right)$. If $\phi: U \rightarrow F$ is a $C^{r}$-Fredholm map with $r>\max (\operatorname{ind}(\phi), 0)$, then the image $\phi(C)$ of its critical set is meager in $F$ (i.e., $\phi(C)$ is expressible as a countable union of sets whose closures contain no interior point of $F)$. Smale [111] has used this to establish a local uniqueness theorem for certain second order nonlinear elliptic differential equations in bounded domains of $R^{n}$ with Dirichlet boundary data.

ExAmple. Suppose $\Phi: U \rightarrow F$ is $C^{r}(r \geqq 1)$ and is a compact map (i.e., $\Phi$ maps bounded subsets of $U$ into relatively compact subsets of $F)$; then at every $x \in U$ the differential $\Phi_{*}(x)$ is a compact linear 
map. (A partial converse of this assertion is known: If for each $x \in U, \Phi_{*}(x)$ is compact and if $\Phi_{*}: U \rightarrow L(E, F)$ is compact, then $\Phi$ is itself compact [117, p. 51].) Now if $\phi: U \rightarrow F$ has the form $x \rightarrow \phi(x)$ $=A(x)+\Phi(x)$ for a Fredholm operator $A \in L(E, F)$, then $\phi$ is itself a Fredholm map with ind $\phi=$ ind $A$.

(E) We will not emphasize complex function theory in Banach spaces (and algebras), except to indicate the following broad lines of development. (1) If $E$ and $F$ are complex Banach spaces, a map $\phi$ : $U \rightarrow F$ is holomorphic if and only if for any finite dimensional complex linear subspace $S$ of $E$ the restriction $\phi: S \cap U \rightarrow F$ is holomorphic. Then we have Cauchy's formula $(k \geqq 0)$

$$
d^{k} \phi(x ; v, \cdots, v)=\frac{k !}{2 \pi i} \int \frac{\phi(x+z v)}{z^{k+1}} d z .
$$

(2) Domains of holomorphy in Banach spaces are studied in [14]. See also Douady [24a] for the theory of analytic spaces. (3) The Teichmüller spaces of Fuchsian groups are represented as bounded domains in complex Banach spaces, and have natural complete Finsler structures relative to which complex differential geometry can be brought to bear; see $\$ 5 \mathrm{G}$ below. (4) Certain manifolds, such as the Grassmannians of complex $p$-planes in complex Hilbert space, have a natural (homogeneous) complex structure and admit a good global complex differential geometry.

(F) There are several variations of the notion of class $C^{r}$ of a map $\phi: U \rightarrow F$ which have applications. For instance, (1) we could require that all differentials $d^{k} \phi: U \rightarrow S L^{k}(E, F)(k \leqq r)$ be uniformly continuous on $U$; that has been useful in establishing differentiability of solutions of certain second order ordinary differential equations [43], [77], [54] and in the theory of Banach Lie groups [74, §6]; (2) we could require that these differentials be continuous in smaller locally convex topologies on $S L^{k}(E, F)$. That is a convenient device in the study of the relationship between closed linear operators and analytic 1-parameter semigroups, using the strong operator topology [50]. It also arises in attempting to impose a differentiable manifold structure on spaces of solutions of differential equations-in such a way that the tangent space at a given solution is the totality of solutions of a linearized problem (relative to that solution), and that these tangent spaces vary differentiably; see [109] for an application.

(G) It is important to extend the theory of $C^{r}$-maps of Banach spaces to maps of more general complete locally convex topological vector spaces-at least to Fréchet and nuclear spaces. (There are many applications awaiting such a theory; e.g., in nonlinear func- 
tional analysis in the Fréchet spaces of $C^{\infty}$ real functions on an open $U \subset R^{n}$; in the Montel spaces of real analytic functions in such $U$ (with their van Hove topology [119]); in a study (undertaken by J. Leslie and R. Abraham) of groups of diffeomorphisms of a compact $C^{\infty}$ or analytic manifold; in the structure theory of certain groups of units of locally convex topological algebras (possessing continuous inverses near the identity)). There seems to be substantial difficulty in developing such a theory in a strictly topological framework: compositions do not behave well, and the inverse function theorem is false, as we will see in the following example. There have been several attempts to circumvent these difficulties; in particular, one by Bastiani [6]. The central idea here is to replace the locally convex topologies of $E$ and $L^{k}(E, F)$ by a different sort of convergence, which still respects algebraic operations and locally convex structures.

ExAmple. The inverse function theorem is false for separable Fréchet spaces, with any reasonable definition of differentiability: Let $E=F$ be the space of all continuous real functions on $R$. Then with the topology of compact convergence $E$ is a multiplicatively convex commutative algebra such that every neighborhood of the function 1 contains functions which assume negative values on $R$, and therefore which do not have inverses. Clearly the exponential map exp: $E \rightarrow E$ is well defined, is injective and carries 0 into 1 ; its differential must be $\exp _{*}(0)=I$. However, no neighborhood of 1 is the image under exp of a neighborhood of 0 . (In contrast, the space of real analytic functions on a compact interval with the van Hove topology is a complete locally convex algebra having continuous inverses near 1 (see [119]); that essential difference between these two algebras was pointed out to me by $\mathrm{C}$. Herz.)

\section{Lie groups and algebras.}

(A) There is a satisfactory local theory relating (Banach) Lie algebras and (Banach) Lie groups, due primarily to G. Birkhoff [9]. A (Banach) Lie algebra is a Banach space $L$ which is a Lie algebra (over $R$ ) relative to a bilinear map $x, y \rightarrow[x, y]$ of $L \times L \rightarrow L$, and such that $|[x, y]| \leqq|x||y|$ for all $x, y \in L$. A (Banach) Lie group germ is a topological group germ modeled on a Banach space, whose group operations are real analytic. (It suffices $[74,87]$ for analyticity that these operations be uniformly $C^{3}$; furthermore, all differentiability hypotheses can be weakened substantially.) As in the finite dimensional case, there is a bijective correspondence $G \rightarrow L(G)$ between ( $B a$ nach) Lie group germs and (Banach) Lie algebras. As usual, the Lie algebra will be identified with the tangent space to the neutral element of the Lie group germ. Homomorphisms of Lie algebras cor- 
respond (via the differential) to local analytic homomorphisms of the Lie group germs; closed Lie subalgebras (resp., ideals) correspond to Lie subgroup (resp., invariant subgroup) germs [9], [27], [66], [74]. If $G$ is a Lie group germ, $K$ a closed invariant subgroup germ which splits in $G$, then $G / K$ has the induced compatible structure of a Lie group germ with Lie algebra $L(G) / L(K)$ [74]. Canonical coordinate charts can be introduced in a neighborhood of the neutral element of a Lie group germ, relative to which group multiplication is expressed by an absolutely convergent series involving the Lie bracket; the associated exponential map defined in a neighborhood of 0 in the Lie algebra carries straight lines through 0 bijectively onto 1-parameter group germs.

In contrast to the finite dimensional case, not every Lie group germ can be enlarged to a global Lie group. A general method of producing examples (set in the framework of Banach Lie algebra cohomology) is given in [118], along with the following criterion for enlargability:

If $L$ is a Banach Lie algebra of a global connected and simply connected Lie group $G$ and $A$ a closed ideal in $L$, then the quotient algebra $L / A$ is the Banach Lie algebra of a global Lie group if and only if $A$ generates (through the exponential map) a closed subgroup of $G$.

(B) ExAmpLe. Let $A$ be an associative Banach algebra with unit 1, and $G(A)$ its group of units (i.e., the elements of $A$ which have multiplicative inverses). Then $G(A)$ is an open subset of $A$, and is a Lie group whose Lie algebra is $A$, with bracket defined by $[u, v]=u v-v u$ for all $u, v \in A$. The analytic map exp: $A \rightarrow G(A)$ is given by the familiar power series $\exp (u)=\sum u^{k} / k !$.

Now take a real Hilbert space $E$, let $A=L(E)=L(E, E)$, (a nonseparable Banach space in general, even if $E$ is separable), and denote by $B$ the closed Lie subalgebra of $A$ consisting of the skew-symmetric operators $\left(u^{*}=-u\right)$. Then $B$ is the Lie algebra of the closed Lie subgroup $O(E)$ of $G L(E)$ of the orthogonal $\left(v^{*} v=1\right)$ automorphisms. If $E$ is infinite dimensional, the exponential map exp: $B$ $\rightarrow O(E)$ is not surjective [96], although $O(E)$ is connected. If $u \in L(E)$ is symmetric, then $\exp (u) \in P(E) \subset G L(E)$, where $P(E)$ denotes the open convex cell of symmetric positive definite $\left(\langle u(x), x\rangle \geqq c|x|^{2}\right.$ for some $c>0$ and all $x \in E)$ automorphisms. Furthermore, the product map induces an analytic diffeomorphism of $O(E) \times P(E)$ onto $G L(E)$; [64, p. 102].

A theorem of Kuiper [61] asserts that if $E$ is any infinite dimensional Hilbert space, then $O(E)$ and hence $G L(E)$ is contractible. (A 
similar result with a smaller topology on $G L(E)$ was given by Dixmier-Douady [24, Lemme 3].) Kuiper's theorem is also valid for Hilbert spaces (separable or not) over the complex and quaternion fields. Recently D. Arlt has shown that $G L\left(c_{0}\right)$ is also contractible; and in contrast A. Douady has found that $G L\left(E \times c_{0}\right)$ is not even connected, where $E$ is a separable Hilbert space of infinite dimension.

The totality of $p$-planes through the origin in a separable infinite dimensional Hilbert space $E$ forms a manifold $B O(p)$ which can be represented as a homogeneous space of the Lie group $O(E)$, and has the homotopy type of the limit space

$$
\lim _{k \rightarrow \infty} O(k+p) / O(k) \times O(p),
$$

where $O(k)=O\left(R^{k}\right)$, of the Grassmannians of $p$-planes in $R^{k+p}$. If $E O(p)$ is the homogeneous space of orthonormal $p$-frames in $E$, then the natural coset map $E O(p) \rightarrow B O(p)$ is an analytic universal principal $O(p)$-bundle. For instance, if we take $p=1$, then $E O(1)$ is the unit sphere in $E$, and $B O(1)$ is the infinite dimensional real projective space; the bundle $E O(1) \rightarrow B O(1)$ can also be realized as the normal 0 -sphere bundle of a 1-codimensional projective subspace of $B O(1)$.

(C) Example. If $E$ is a separable infinite dimensional Hilbert space and $C(E)$ is the totality of compact linear endomorphisms of $E$, then $C(E)$ is the only closed proper bilateral ideal of $L(E)$ (as an associative Banach algebra). The set $G C(E)$ of all elements of $G L(E)$ of the form $1+u$, where $u \in C(E)$, is an invariant closed Lie subgroup whose Lie algebra is $C(E) ; G C(E)$ has precisely two components.

By means of an orthonormal base $\left(e_{k}\right)_{k \geqq 1}$ in $E$ we have the imbeddings $G L\left(R^{n}\right) \rightarrow G L\left(R^{n+1}\right) \rightarrow G C(E)$ for all $n \geqq 0$; it is known [90], [115] that these inclusions induce a homotopy equivalence

$$
\lim _{n \rightarrow \infty} G L\left(R^{n}\right) \rightarrow G C(E) .
$$

The left member is the direct limit of the groups $G L\left(R^{n}\right)$; its homotopy properties are now well known through the periodicity theorems of Bott [12]. Palais [90] goes on to establish the corresponding result for certain other Banach algebras of operators on $E$ (contained algebraically in $C(E)$ ), including the algebras of $L^{p_{\text {-operators }}}$ $(1 \leqq p<\infty)$.

Let $G_{r} C(E)=\left\{v \in G C(E): v\left(e_{k}\right)=e_{k}\right.$ for all $\left.k>r\right\}$, and $G^{r} C(E)$ $=\left\{v \in G C(E): v\left(e_{k}\right)=e_{k}\right.$ for all $\left.k \leqq r\right\}$. The homogeneous spaces $V_{r}=G C(E) / G^{r} C(E)$ and $V^{r}=G C(E) / G_{r} C(E)$ are called the Stiefel manifolds of $r$-frames and $r$-coframes of $C(E)$-vectors. We have the successive fibrations 


$$
\begin{aligned}
I & =V_{0} \leftarrow V_{1} \leftarrow V_{2} \leftarrow \cdots . \\
G C(E) & =V^{0} \rightarrow V^{1} \rightarrow V^{2} \rightarrow \cdots .
\end{aligned}
$$

Every $V_{r}$ is contractible, and $V^{r}$ has the homotopy type of $V^{r}\left(\lim _{n \rightarrow \infty} L\left(R^{n}\right)\right)$ [75].

If $p: L(E) \rightarrow L(E) / C(E)$ is the coset map and $G=G[L(E) / C(E)]$ is the group of units of the indicated quotient algebra, then $p^{-1} G$ is identified with the set $\mathfrak{F}$ of Fredholm operators of $E$. The map $p: \mathfrak{F} \rightarrow G$ is a Serre fibration with contractible fibres, and therefore [52; see also $\S 4 \mathrm{~A}$ below] $p$ is a homotopy equivalence. Also, the identity component of $G$ is isomorphic to $G L(E) / G C(E)$; that latter group can then be used as the classifying space $B O$ for real vector bundles. The following theorem is due to Jänich [52]: Using composition of operators as addition and a multiplication derived from the tensor product, $\mathfrak{F}$ has up to homotopy the structure of a commutative ring with unit, and those operations are continuous.

For any compact space $X$ the totality $[X, \mathfrak{F}]$ of homotopy classes of maps $X \rightarrow \mathfrak{F}$ is a commutative ring with unit. There is a natural ring isomorphism $[X, \mathfrak{F}] \rightarrow K O(X)=[X, Z \times B O]$ of $[X, \mathfrak{F}]$ onto the ring $K O(X)$ of virtual real vector bundles over $X$. Here $\boldsymbol{Z}$ denotes the ring of integers. The isomorphism is constructed as a generalization of the notion of index of a Fredholm operator; the proof uses Kuiper's theorem on the contractibility of $G L(E)$. See [12] for the definitions and properties of the functors $K O$ and $B O$ (=the classifying space of the group $\lim _{n \rightarrow \infty} G L\left(R^{n}\right)$; or $\left.B O=\lim _{p \rightarrow \infty} B O(p)\right)$. Completely analogous assertions are valid for the complex case; and an appropriate assertion can also be made for the case of the quaternion field.

(D) Example [31]. The following construction admits many variations of the sort considered in $\S 6$ below. Let $G$ be a connected Lie group of finite dimension with Lie algebra $L(G)$, equipped with some inner product. We denote by $E(G)$ the totality of absolutely continuous paths on $G$ starting at the neutral element $e$ and having square integrable tangent fields; thus $E(G)=L_{1}^{2}(I, 0 ; G, e)$ in the notation of $\S 6 \mathrm{D}$ below. With group operations defined pointwise $E(G)$ has the structure of a contractible Lie group modeled on a separable Hilbert space, and the Serre map $p: E(G) \rightarrow G$ defined by $p(x)=x(1)$ is a Lie epimorphism whose kernel $\Omega(G)$ can be viewed as the Lie group of loops (of the indicated class) of $G$; furthermore, $\operatorname{codim}(E(G), \Omega(G))=\operatorname{dim} G$. Its Lie algebra $L(E(G))=E(L(G))$, and the kernel of the differential $p_{*}: L(E(G)) \rightarrow L(G)$ is just $L(\Omega(G)$ ). (In the special case that $G$ is compact and semisimple and we take the negative of the Killing form on $L(G)$ for its inner product, then 
$\langle[x, y], z\rangle=\langle x,[y, z]\rangle$ for all $x, y, z \in L(G)$; however, we do not have the corresponding relation in $L(E(G))$, so that $L(E(G))$ should be viewed as a Banach, not as a Hilbert, Lie algebra.)

Now suppose that $K$ is a closed subgroup of $G$, and let $\pi: G \rightarrow G / K$ $=B$ be the (left) coset map. If $E(G, K)=p^{-1}(K)$ and $\lambda=\pi \circ p$ then $E(G, K)$ is a closed Lie subgroup of $E(G)$, and $\lambda: E(G) \rightarrow B$ is an analytic universal principal $E(G, K)$-bundle; thus $E(G, K)$ is a Lie group serving as the loop space of $B$.

(E) For further applications relating Banach algebraic and differential topological ideas we refer to

(1) Dixmier-Douady [24] and Tomiyama-Takesaki [116] for studies of $C^{*}$-algebras and continuous fields of Hilbert spaces by the methods of cohomology and fibre bundles;

(2) Royden [103] for a theorem of Arens-Royden relating the cohomology of the spectrum of a commutative Banach algebra to its group of units;

(3) Wood [121] for a proof and generalization of the Bott periodicity theorem in the setting of Banach algebras.

(4) Myers [85] and Pursell-Shanks [95] for theorems giving the structure of a compact $C^{r}$-manifold in terms of its algebra of $C^{r}$ functions.

\section{Manifolds and fibre bundles.}

(A) A manifold ( $C^{0}$-manifold) modeled on a locally convex topological vector space $E$ is a Hausdorff topological space $X$ such that with each point $x \in X$ there exist a neighborhood $U_{x}$ and a homeomorphism $\theta_{x}$ of $U_{x}$ onto a convex open subset of $E$ with $\theta_{x}(x)=0$; the pair $\left(\theta_{x}, U_{x}\right)$ is called a chart of $X$ centered at $x$. Clearly $X$ is completely regular and locally contractible; in particular, every component of $X$ is open. Assume that $E$ is metrizable; then applications of theorems of A. Stone and Y. Smirnov show that $X$ is metrizable when and only when $X$ is paracompact. If every component of $X$ satisfies the second axiom of countability, then $X$ is metrizable [92]. If $X$ is paracompact, then the components of $X$ satisfy the second axiom of countability if and only if $E$ does [75].

Hypothesis. Henceforth we suppose that $X$ is a paracompact manifold modeled on a metrizable locally convex topological vector space $E$.

A theorem of Dugundji [25] asserts that any convex set in a locally convex topological vector space is an absolute retract. (A space $U$ (usually assumed to be metrizable) is an absolute retract if for any metrizable space $M$, closed subset $A \subset M$, and continuous map 
$f: A \rightarrow U$ there is a continuous extension to a map $M \rightarrow U$; if every $f$ can be extended to a neighborhood of $A$ in $M$, then we say that $U$ is an absolute neighborhood retract.) Dugundji's theorem insures furthermore that we can extend any $f: A \rightarrow U$ so that the extension has its image in the convex envelope of $f(A)$. General principles and a theorem of $\mathrm{O}$. Hanner then imply the following result (proved by the author in connection with his version of the Lefschetz fixed point theorem ( $\$ 11 \mathrm{D}$ below), utilizing a method of W. Hurewicz. An independent proof appears in [92]).

If $X$ is a paracompact manifold modeled on a metrizable locally convex topological vector space, then $X$ is an absolute neighborhood retract. $X$ is contractible if and only if $X$ is an absolute retract.

It is a consequence of a theorem of J. H. C. Whitehead that if $X$ and $Y$ are two such manifolds and $f: X \rightarrow Y$ a continuous map inducing isomorphisms $f_{*}: \pi_{i}(X) \rightarrow \pi_{i}(Y)$ of the homotopy groups for all $i \geqq 0$, then $f$ is a homotopy equivalence. Theorems of O. Hanner and J. H. C. Whitehead [78] imply that if $X$ is separable, then it has the homotopy type of a countable locally finite simplicial polyhedron.

If $X$ is given a metric, then $X$ can be isometrically imbedded as a closed subset of a normed linear space, having a neighborhood $U$ with $X$ as a retract (i.e., there is a continuous map $f: U \rightarrow X$ which is the identity on $X$ ) [4]. If the model $E$ is complete, then we can always so imbed $X$ topologically as a closed subset of a Banach space. In particular, the topology of $X$ can be given by a complete metric, so that $X$ is a Baire space (i.e., a space such that any countable union of closed sets having no interior points has no interior point). $X$ is locally compact if and only if its model $E$ has finite dimension, by a theorem of F. Riesz; thus a component of $X$ is expressible as a countable union of compact sets when and only when $X$ is finite dimensional.

(B) Let $X$ be a manifold modeled on a Banach space $E$. A $C^{r_{-}}$ structure on $X(1 \leqq r \leqq \infty)$ is a subsheaf of the sheaf of continuous $E$ valued maps on $X$ which is locally isomorphic (through charts) to the sheaf of $E$-valued $C^{r}$-maps on $E$; similarly for the notions of real and complex analytic structures. That amounts to saying that a $C^{r}-$ structure is a maximal covering of $X$ by charts such that all maps (between the indicated open subsets of $E$ )

$$
\theta_{x^{\prime}} \circ \theta_{x}^{-1}: \theta_{x}\left(U_{x} \cap U_{x^{\prime}}\right) \rightarrow \theta_{x^{\prime}}\left(U_{x} \cap U_{x^{\prime}}\right)
$$

are $C^{r}$. A $C^{r}$-manifold is a $C^{0}$-manifold with a specific $C^{r}$-structure. For $r>0$ we have the related notion of $C^{r}$-manifold with boundary; 
however, there is not a suitable definition in case $r=0$ and the manifold has infinite dimension, due to the absence of invariance of domain (See §11B below.) Indeed, Klee [56] has constructed a homeomorphism of a separable Hilbert space onto a closed half-space. A $C^{r}$-map $f: X \rightarrow Y$ of $C^{r}$-manifolds is a map which respects the $C^{r}$ structures: For any $C^{r}$-charts $(\theta, U),(\chi, V)$ on $X, Y$ the composition $\chi \circ f \circ \theta^{-1}: \theta(U) \rightarrow \chi(V)$ is a $C^{r}$-map. A bijective $C^{r}$-map $f$ with $C^{r}$ inverse is a $C^{r}$-diffeomorphism.

If $E_{0}$ is a closed linear subspace of the model $E$ and $A$ a closed subset of the $C^{r}$-manifold $X$ such that for every $x \in A$ there is an $x$ centered chart $(\theta, U)$ of $X$ such that $\theta$ maps $U \cap A$ homeomorphically on to $\theta(U) \cap E_{0}$, then these restrictions determine a $C^{r}$-structure on $A$, and $A$ is called a closed $C^{r}$-submanifold of $X$. A $C^{r}$-imbedding (resp., $a C^{r}$-immersion) $f: X \rightarrow Y$ is a $C^{r}$-map which is a $C^{r}$-diffeomorphism (resp., locally a $C^{r}$-diffeomorphism) on to a closed submanifold of $Y$. Say that the imbedding or immersion splits if its model $E_{0}$ is a direct summand of $E$ (and be advised that some authors [2], [64] incorporate the splitting requirement into their definition of closed submanifold). There is a concept dual to a split $C^{r}$-immersion: A $C^{r}$ map $f: X \rightarrow Y$ is called a split $C^{r}$-local fibration if for all $x \in X$ there are $C^{r}$-charts $(\theta, U),(\chi, V)$ centered at $x, f(x)$, where $\theta: U \rightarrow U_{1} \times U_{2}$ maps $U$ onto the product of two open sets in a product representation $E_{1} \times E_{2}=E$ of the model of $X$, such that the composition $\chi \circ f \circ \theta^{-1}$ : $U_{1} \times U_{2} \rightarrow \chi(V)$ is a projection.

The following generalization of Whitney's imbedding theorem has been proved by McAlpin [75]; see also [18]:

Every separable $C^{r}$-manifold modeled on a separable Hilbert space can be split $C^{r}$-imbedded as a closed submanifold of a separable Hilbert space.

(C) There is a theory of $C^{r}$-vector bundles whose fibres are Banach spaces; its broad outline follows that of the finite dimensional case [64]. Of course, we must check certain technical aspects: (1) A $C^{0}$-vector bundle whose fibres are Banach spaces over a $C^{0}$-manifold is metrizable [92]. (2) In arguments involving partitions of unity we should suppose that the base manifolds are modeled on $C^{r}$-smooth Banach spaces; e.g., the sheaf of $C^{r}$-sections of a $C^{r}$-vector bundle over a $C^{r}$-manifold with $C^{r}$-smooth model is soft (i.e., any section over a closed subset of the base admits an extension over all the base). (3) In dealing with sequences

$$
0 \rightarrow A \stackrel{\phi}{\rightarrow} B \stackrel{\psi}{\rightarrow} C \rightarrow 0
$$


of vector bundles over $X$ we need a special notion of exactness: Say that this sequence is exact if the restriction $\phi: A_{x} \rightarrow B_{x}$ of the bundle map $\phi$ to the fibre over every point $x \in X$ is a continuous injection, the restriction $\psi: B_{x} \rightarrow C_{x}$ is a continuous surjection with $\operatorname{Ker}(\psi)$ $=\operatorname{Im}(\phi)$, and every $\phi\left(A_{x}\right)$ is a direct summand of $B_{x}$. This last requirement insures a suitable local product structure for the maps $\phi, \psi$. (4) There are many different tensor products of vector bundles, depending on the choice of topological tensor product in the fibres.

(D) Let $X$ be a $C^{r}$-manifold $(r \geqq 1)$ modeled on a Banach space $E$. Then as in the finite dimensional case [114] the differentials $\left(\theta_{x^{\prime}} \circ \theta_{x}^{-1}\right)_{*}$ of the coordinate changes (2) define transition maps of a principal bundle $G L(X) \rightarrow X$ with structural group $G L(E)$, called the principal bundle of $X . G L(X)$ has the structure of $C^{r-1}$-manifold. From Kuiper's theorem ( $\S 3 \mathrm{~B})$ we obtain the result:

Every paracompact infinite dimensional $C^{r}$-manifold $(r \geqq 1) X$ modeled on a separable Hilbert space is $C^{r-1}$-parallelizable. I.e., its principal bundle is $C^{r-1}$-isomorphic to the product bundle $X \times G L(E)$.

From the natural action of $G L(E)$ on $E$ we obtain the associated tangent vector bundle $\pi: T(X) \rightarrow X$; each fibre $\pi^{-1}(x)=X(x)$, called the tangent vector space to $X$ at $X$, is a Banach space-but does not have a preferred norm in general, even if $E$ does. A $C^{r}$-map $f: X \rightarrow Y$ induces through its differential a $C^{r-1}$-map $T(f): T(X) \rightarrow T(Y)$ which is linear and continuous on each fibre. Similarly for the various tensor bundles of $X$.

(E) If $f: X \rightarrow Y$ is a split $C^{r}$-immersion, then the sequence

$$
0 \rightarrow T(X) \rightarrow f^{-1} T(Y) \rightarrow f^{-1} T(Y) / T(X) \rightarrow 0
$$

is exact, where $f^{-1} T(Y) \rightarrow X$ is the induced vector bundle. If $f: X \rightarrow Y$ is a split $C^{r}$-local fibration, then there is a subbundle $A \rightarrow X$, called the bundle along the fibres, which is the kernel of the differential of $f$, such that the sequence

$$
0 \rightarrow A \rightarrow T(X) \rightarrow f^{-1} T(Y) \rightarrow 0
$$

is exact. It may not happen that these exact sequences themselves split as $C^{r-1}$-bundles; however, they do if $X$ is modeled on a $C^{r_{-}}$ smooth Banach space. In that case a choice of splitting in (3) identifies $f^{-1} T(Y) / T(X)$ with a subbundle of $f^{-1} T(Y)$ supplementary to $T(X)$, called a transverse bundle of $X$ in $Y$. Similarly, a splitting in (4) identifies $f^{-1} T(Y)$ with a supplement of $A$ in $T(X)$ called a horizontal subbundle.

An application [32], [64] of the inverse function theorem gives the 
following result (as formulated in the finite dimensional case by $R$. Thom).

Let $f: X \rightarrow Y$ be a $C^{r}$-map $(r>1)$ of manifolds modeled on Banach spaces. Let $B$ be a closed split imbedded $C^{r}$-submanifold of $Y$ on which $f$ is transversal (i.e., for every $x \in f^{-1}(B)$ the differential $f_{*}(x): X(x)$ $\rightarrow Y(f(x))$ is transversal to $B(f(x))$ in the sense of $\$ 1 C)$. Then $A=f^{-1}(B)$ is a closed split imbedded $C^{r}$-submanifold of $X$.

If $\operatorname{codim}(Y, B)=q<\infty$, then $\operatorname{codim}(X, A)=q<\infty$. Also, if $\operatorname{dim} B$ $<\infty$ and $f$ is a Fredholm map of connected separable manifolds, then $\operatorname{dim} A-\operatorname{dim} B=\operatorname{ind}(f) ;$ Smale [111] has shown (as a consequence of results of $\S 2 \mathrm{D})$ that for any Fredholm $C^{r}$-map $f: X \rightarrow Y$ there is a $C^{1}$ approximation to the imbedding $B \rightarrow Y$ on which $f$ is transversal, provided that $r>\max (0, \operatorname{ind}(f)+\operatorname{dim} B)$.

EXAMpLE. The diagonal $\Delta=\{(y, y) \in Y \times Y\}$ is a closed split imbedded submanifold of $Y \times Y$, with transverse bundle isomorphic to $T(Y)$. Two maps $f_{1}, f_{2}: X \rightarrow Y$ are transversal if the product map $f_{1} \times f_{2}: X \times X \rightarrow Y \times Y$ is transversal on $\Delta$. Then $\left(f_{1} \times f_{2}\right)^{-1}(\Delta)$ is a closed split imbedded submanifold of $X \times X$; its intersection with the diagonal of $X \times X$ is identified with the coincidence set $\Gamma\left(f_{1}, f_{2}\right)$ $=\left\{x \in X: f_{1}(x)=f_{2}(x)\right\}$. This situation occurs frequently in applications; for instance, let $\xi: Y \rightarrow X$ be a $C^{r}$-vector bundle and $\sigma$ a $C^{r}$ section (i.e., a $C^{r}$-map $\sigma: X \rightarrow Y$ such that $\xi \circ \sigma(x)=x$ for all $x \in X$ ). If $\zeta$ is the zero section, then $\Gamma(\sigma, \zeta)$ is just the locus of zeros of $\sigma$; the condition that $\sigma, \zeta$ be transversal is a nondegeneracy hypothesis on $\sigma$.

(F) If $X$ is a $C^{r}$-manifold $(r \geqq 2)$ modeled on a Banach space, then its tangent bundle $T(X)$ is a $C^{r-1}$-manifold. The totality $C^{r-1}(T(X))$ of $C^{r-1}$ sections (i.e., of $C^{r-1}$ maps $v: X \rightarrow T(X)$ such that $\pi \circ v(x)=x$ for all $x \in X$ ) forms a vector space, with algebraic operations defined pointwise; these sections are called vector fields on $X$. The Poisson bracket $[u, v]$ of two vector fields is defined as an element of $C^{r-2}(T(X))$; in particular, if $r=\infty$, then the bracket defines a Lie algebra structure on $C^{\infty}(T(X))$.

The trajectories of a vector field $v \in C^{r-1}(T(X))$ are the solutions of the differential equation

$$
\begin{aligned}
\frac{d \phi_{t}(x)}{d t} & =v\left(\phi_{t}(x)\right), \\
\phi_{0}(x) & =x,
\end{aligned}
$$

defined for some maximal interval $\alpha_{x}<t<\beta_{x}$ of $R$ containing 0 . The existence, uniqueness, and differentiability with respect to the initial 
conditions of such solutions follow from standard methods [23, Chapter X]; [64, Chapter IV]. The set $D(v)=\{(t, x) \in R \times X$ : $\left.\alpha_{x}<t<\beta_{x}\right\}$ is an open neighborhood of $0 \times X$, and on it the map $(t, x) \rightarrow \phi_{t}(x)$ defines a 1-parameter group germ of $C^{r-1}$-diffeomorphisms of $X$. It is a matter of basic importance to know when $D(v)$ $=R \times X$; i.e., when the trajectories are full. That is a question of the growth of $v$, and can be best described in terms of a Finsler or Riemannian structure (see $\S 5$ ); in the case that $X$ is a separable complete Riemannian manifold good criteria are given in [68]; e.g., every trajectory of finite length is full. See also $\$ 8$ below for the case of gradient fields.

The integrability theorem of Frobenius is valid for $C^{r}$-manifolds $(r \geqq 2)$ modeled on Banach spaces [23], [64], [73, Part II]; we state the result for $r=\infty$ :

Let $S$ be a $C^{\infty}$-vector subbundle of $T(X)$ for which every fibre $S_{x}$ is a direct summand of $X(x)$. Then the space $C^{\infty}(S)$ of sections is a Lie subalgebra of $C^{\infty}(T(X))$ if and only if $S$ is integrable; i.e., $X$ is a leaved manifold having $S$ as the tangent bundle of the leaved structure. A split $C^{\infty}$-local fibration $f: X \rightarrow Y$ provides an example of this situation; see $\$ 5 \mathrm{G}$.

(G) Let $X$ be a $C^{\infty}$-manifold modeled on a separable Hilbert space, and $\Lambda^{p} T^{*}(X) \rightarrow X$ its vector bundle of $p$-covectors; its fibre over $x$ can be identified with $A L^{p}(X(x), R)$, the Banach space of continuous alternating $p$-linear forms on $X(x)$. Denote by $\Lambda T^{*}(X)$ the direct sum of these bundles for all $p \in \boldsymbol{Z}$; then the vector space $C^{\infty}\left(\Lambda T^{*}(X)\right)$ of $C^{\infty}$-sections, which are called differential forms on $X$, is a graded associative algebra (over $R$ ) with unit, and commutative (in the sense that $\phi \wedge \psi=(-1)^{p q} \psi \wedge \phi$ if $\phi \in C^{\infty}\left(\Lambda^{p} T^{*}(X)\right), \psi \in C^{\infty}\left(\Lambda^{q} T^{*}(X)\right)$. There is a linear map $d: C^{\infty}\left(\Lambda^{p} T^{*}(X)\right) \rightarrow C^{\infty}\left(\Lambda^{p+1} T^{*}(X)\right)$, called the exterior differential, which is a derivation $\left(d(\phi \wedge \psi)=d \phi \wedge \psi+(-1)^{p} \phi\right.$ $\wedge d \psi$ with $\phi, \psi$ as above), which has square zero $(d(d \phi)=0)$, and which assigns to each function $\phi \in C^{\infty}\left(\Lambda^{0} T^{*}(X)\right)$ its differential. The space $\mathfrak{F}^{*}(X)=\operatorname{Ker}(d) / \operatorname{Im}(d)$ is therefore a graded commutative algebra with unit, called the de Rham cohomology algebra of $X$.

A $p$-simplex of class $C^{\infty}$ of $X$ is a $C^{\infty}$-map $s: \Delta^{p} \rightarrow X$ of a neighborhood of an oriented rectilinear $p$-simplex $\Delta^{p}$ of $R^{p}$; a $p$-chain of class $C^{\infty}$ of $X$ is a finite linear combination (over $R$ ) of such $p$-simplexes, and the boundary $\partial c$ of a $p$-chain $c$ is defined as usual in algebraic topology. The $p$-chains constitute a vector space $S_{p}(X)$, and $\partial: S_{p}(X)$ $\rightarrow S_{p-1}(X)$ is a linear map satisfying $\partial(\partial c)=0$ for all $c \in S_{p}(X)$; if $S(X)$ denotes the direct sum of the $S_{p}(X)$ for all $p \in Z$, then $\partial$ is an 
endomorphism of $S(X)$, and we define the graded vector space $H(X)=\operatorname{Ker}(\partial) / \operatorname{Im}(\partial)$, the real homology group of $X . H(X)$ is a homology invariant of the space $X$.

For any $p$-form $\phi \in C^{\infty}\left(\Lambda^{p} T^{*}(X)\right)$ and $p$-chain $c \in S_{p}(X)$ we define the Riemann integral of $\phi$ over $c$ in the familiar manner, using the transformation of integral formula. Then we have Stokes' theorem

$$
\int_{c} d \phi=\int_{\partial c} \phi
$$

so that the integral induces a bilinear pairing

$$
\mathcal{F}^{p}(X) \times H_{p}(X) \rightarrow R .
$$

Otherwise said, each de Rham cohomology class $(\phi) \in \mathcal{F}^{p}(X)$ thereby defines a linear form on the homology group $H_{p}(X)$, and thus a cohomology class in $H^{p}(X)=\operatorname{Hom}\left(H_{p}(X), R\right)$. We obtain the following generalization of de Rham's theorem [29]:

Let $X$ be a separable $C^{\infty}$-manifold modeled on a separable Hilbert space. Then integration defines a degree-preserving algebra isomorphism of the de Rham cohomology algebra $\mathfrak{H}^{*}(X)$ onto the real singular cohomology algebra $H^{*}(X)$.

Several specific representations illustrating this theorem are given in [31], [118]; the latter paper uses 2-forms on certain Banach Lie groups to construct central extensions (for the purpose described in $\S 3 \mathrm{~A})$.

\section{Differential geometry.}

(A) Certain aspects of local differential geometry (tensor analysis, linear connections and covariant derivatives, geodesics, curvature forms and sectional curvature, leaved structures) in open sets of Banach spaces made their appearance in one form or another many years ago; we refer to [77], [55], [65] and the references in [77] for various treatments. Practically no global differential geometry has been developed for manifolds modeled on Banach spaces other than Hilbert spaces; however, it seems likely that infinite dimensional Finsler geometry will play an important role in the future.

The foundations of modern differential geometry center around the theory of integrable $G$-structures (for arbitrary Lie groups $G$ ). We will not deal in that generality here; however, let us remark in passing that there is an interesting aspect of the general theory peculiar to the infinite dimensional case: For a $C^{r}$-manifold $X$ modeled on a Banach space $E$ we take $G=G C(E)$, as in $\S 3 \mathrm{C}$. Then an integra- 
ble $G C(E)$-structure on $X$ is determined by a covering of $X$ by coordinate charts, the differentials of whose coordinate changes (2) lie in $G C(E)$.

We now examine certain elementary properties-local and globalof Riemannian geometry, thus taking $G=O(E)$; the integrability question does not arise. For the most part, the local theory follows the finite dimensional case; the global theory is substantially different. In this section it is less important to be precise about the differentiability classes of manifolds and bundles; we therefore of ten take $r=\infty$.

(B) Let $X$ be a $C^{\infty}$-manifold modeled on a Banach space $E$, and $\xi: V \rightarrow X$ a $C^{\infty}$-vector bundle whose fibres are Hilbert spaces. We can then form the vector bundle $S L^{2}(V, R) \rightarrow X$ whose fibre over any point $x \in X$ is the space of continuous symmetric bilinear forms on the fibre $\xi^{-1}(x)=V_{x}$. A $C^{\infty}$-Riemannian structure on $\xi$ is a $C^{\infty}$-section of $S L^{2}(V, R) \rightarrow X$ which is positive definite at every point $x$, and which as an inner product on $V_{x}$ determines its Hilbert space structure. If the model $E$ of $X$ is $C^{\infty}$-smooth, then any such vector bundle $\xi$ admits a $C^{\infty}$-Riemannian structure; furthermore, these Riemannian structures on $\xi$ correspond bijectively to reductions of the general linear group of the fibre to its orthogonal group [64, Chapter VII].

Let $C^{\infty}(V)$ denote the vector space of $C^{\infty}$-sections of $\xi$. A covariant differential on $\xi$ is a map $\nabla: C^{\infty}(T(X)) \times C^{\infty}(V) \rightarrow C^{\infty}(V)$, written $\nabla(u, \phi)=\nabla_{u} \phi$, such that (1) the map $u \rightarrow \nabla_{u} \phi$ is $C^{\infty}(X \times R)$-linear for each $\phi \in C^{\infty}(V)$; (2) the map $\phi \rightarrow \nabla_{u} \phi$ is $R$-linear for each $u \in C^{\infty}(T(X))$; (3) $\nabla_{u}(\gamma \phi)=\left(\nabla_{u} \gamma\right) \phi+\gamma \nabla_{u} \phi$ for each $\phi \in C^{\infty}(V), \gamma \in C^{\infty}(X \times R)$, where $\nabla_{u} \gamma=d \gamma \cdot u$. (A covariant differential on $\xi$ is equivalent to a linear connection on $\xi$.) The curvature of $\nabla$ is the map $R: C^{\infty}(T(X))$ $\times C^{\infty}(T(X)) \times C^{\infty}(V) \rightarrow C^{\infty}(V)$ given by

$$
R\left(u_{1}, u_{2}\right) \phi=\nabla_{u_{1}} \nabla_{u_{2}} \phi-\nabla_{u_{2}} \nabla_{u_{1}} \phi-\nabla_{\left[u_{1}, u_{2}\right]} \phi .
$$

We have associated differentials for the adjoint bundle $\xi^{*}$, for tensor powers of $\xi$ and of $\xi^{*}$ and for direct sums and tensor products of bundles with covariant differentials.

A $C^{\infty}$-Riemannian bundle $\xi: V \rightarrow X$ consists of a pair $(\nabla, g)$, where $\nabla$ is a covariant differential on $\xi, g \in C^{\infty}\left(S L^{2}(V, R)\right)$ is a Riemannian structure, and $\nabla_{u} g=0$ for all $u \in C^{\infty}(T(X))$.

(C) Suppose that the $C^{\infty}$-manifold $X$ is modeled on a separable Hilbert space $E$. A Riemannian structure on $X$ is a $C^{\infty}$-Riemannian structure $g$ on its tangent bundle $\pi: T(X) \rightarrow X$ (and we say that $X$ is a Riemannian manifold); these are in natural bijective correspondence with the $C^{\infty}$-sections of the associated bundle of the principal 
bundle $G L(X) \rightarrow X$ whose fibre is the cell $P(E)=G L(E) / O(E)$. The value of $g$ at $x$ is sometimes written $g_{x}(u \otimes v)=\langle u, v\rangle_{x}$ for all $u, v \in X(x)$. The fundamental theorem of Riemannian geometry is the following:

Let $(X, g)$ be a Riemannian manifold. Then there is a unique covariant differential $\nabla$ (called the Levi-Civita differential of $(X, g)$ ) on the tangent bundle making it into a Riemannian bundle, for which the torsion

$$
T\left(u_{1}, u_{2}\right)=\nabla_{u_{1}}\left(u_{2}\right)-\nabla_{u_{2}}\left(u_{1}\right)-\left[u_{1}, u_{2}\right]
$$

vanishes for all $u_{1}, u_{2} \in C^{\infty}(T(X))$.

The Riemannian structure $g$ determines a metric $\rho$ on $X$ which is compatible with its given topology; namely, given any two points $x_{0}, x_{1}$ in the same component of $X$ we define the distance

$$
\rho\left(x_{0}, x_{1}\right)=\inf \left\{\int\left|\gamma^{\prime}(t)\right|_{\gamma(t)} d t\right\}
$$

over all piecewise differentiable paths $\gamma: I \rightarrow X$ joining $x_{0}$ to $x_{1}$, where $\left|\gamma^{\prime}(t)\right|_{\gamma(t)}$ denotes the $g$-norm of the tangent vector $\gamma^{\prime}(t) \in X(\gamma(t))$. A consequence of McAlpin's imbedding theorem is the following:

Every separable $C^{\infty}$-manifold modeled on a separable Hilbert space admits a complete Riemannian structure g; i.e., the metric (7) determined by $\mathrm{g}$ is complete (in the sense of Cauchy sequences).

More generally, we say that a $C^{1}$-manifold $X$ modeled on a Banach space is a Finsler manifold if we have a continuous assignment of a norm to each tangent space which is compatible with its Banach space structure, locally uniformly on $X$; then, as above, every $X$ admits a Finsler structure which determines a metric on $X$ compatible with its topology [89], [91]; see also [67].

(D) Let $X$ be a Riemannian manifold, and $x \in X$. Given two orthonormal vectors $u, v \in X(x)$ we define the sectional curvature of the plane direction $u \wedge v$ (exterior product of the vectors) determined by them through the formula

$$
K(u \wedge v)=\left\langle R_{x}(v, u) u, v\right\rangle_{x}
$$

where $R_{x}$ denotes the curvature (5) of the Levi-Cività differential at $x$. An immediate extension of the Clifford-Klein theorem [75], [77] gives:

If $X$ is a simply connected complete Riemannian manifold of infinite 
dimension and constant sectional curvature, then $X$ is isometric to the infinite dimensional elliptic, parabolic, or hyperbolic space.

In particular, if $G$ is a finite group of isometries operating freely and differentiably on the unit sphere $S$ in infinite dimensional separable Hilbert space, then the orbit space $S / G$ is a Riemannian manifold of constant positive curvature representing the classifying space $B G$ for $G$.

(E) If $X$ is a Riemannian manifold, then its Levi-Cività differential $\nabla$ determines the notion of parallel transport along a $C^{\infty}$-path $\gamma: I \rightarrow X$; in particular $\gamma$ is a geodesic (segment) if its tangent field $\gamma^{\prime}$ is parallel along $\gamma$; i.e., $\gamma$ satisfies the Euler equation $\nabla_{\gamma^{\prime}}\left(\gamma^{\prime}\right)=0$ along $I$. All geodesics on a complete Riemannian manifold are full.

For every point $x$ of a complete Riemannian manifold $X$ there is a $C^{\infty}$-map $\exp _{x}: X(x) \rightarrow X$ defined by letting $\exp _{x}(u)$ be the endpoint of the geodesic segment emanating from $x$ and determined in length and direction by the vector $u$. Local properties of $\exp _{x}$ produce the result: Every point of $X$ has a convex neighborhood; i.e., a neighborhood $U$ such that any two points in $U$ can be joined by a unique geodesic segment lying in $U$ whose length is the distance between its endpoints. Furthermore, we have the following generalization of a theorem of G. de Rham [75]:

Any separable Riemannian manifold $X$ admits a countable open cover $\mathfrak{U}$ by sets all of whose intersections are convex.

It follows upon application of a theorem of J. Leray that the cohomology of $X$ is canonically isomorphic to that of the nerve of $\mathfrak{U}$. On the other hand, and in defiance of the fact (Section 4A) that $X$ (if separable) has the homotopy type of a locally finite polyhedron, we have the theorem of Corson [20]: There is no locally finite cover of a reflexive Banach space of infinite dimension by bounded convex sets.

Another consequence of the existence of convex neighborhoods is the following:

If $X$ is a closed $C^{\infty}$-submanifold of the Riemannian manifold $Y$, then there is a tubular neighborhood $U$ of $X$ in $Y$. Furthermore, the diffeomorphism type of $U$ is unique.

Thus $U$ is diffeomorphic to the normal vector bundle of $X$ in $Y$, and $X$ is a strong $C^{\infty}$-deformation retract of $U$. For a discussion of the existence and uniqueness of tubular neighborhoods in $C^{r}$-manifolds modeled on other Banach spaces, see [64, Chapter IV].

(F) Let $X$ be a connected complete Riemannian manifold. It is not 
known whether the map $\exp _{x}: X(x) \rightarrow X$ is surjective in general. The situation is complicated [47] in the infinite dimensional case by the fact that the differential of $\exp _{x}$ may be neither injective nor surjective at a conjugate point of $x$. Furthermore, it is not always possible to join two points by a geodesic segment of minimum length. A simple example [46], [75] is obtained by studying the geodesics joining the axis points of an infinite dimensional ellipsoid in Hilbert space; such a manifold is contractible and has strictly positive sectional curvatures at every point.

We do have a generalization of the Cartan-Hadamard theorem, due independently to McAlpin and Grossman [46], [75]; see also [47] for a variant:

If $X$ is a connected complete Riemannian manifold of nonpositive sectional curvature, then for any point $x \in X$ the map $\exp _{x}: X(x) \rightarrow X$ is a surjective $C^{\infty}$-covering.

In particular, if $X$ is simply connected, then it is diffeomorphic to Hilbert space; and there is a unique geodesic segment joining any two points, whose length equals the distance between them.

(G) For a surjective $C^{1}$-map $f: X \rightarrow Y$ of $C^{1}$-manifolds modeled on Banach spaces, we have described in Section $4 \mathrm{~B}$ conditions on the differential of $f$ which imply that $f$ is a split $C^{1}$-local fibration. We now impose differential geometric restrictions sufficient to insure that $f$ is a global fibration. Let $\alpha$ and $\beta$ be Finsler structures on $X$ and $Y$. If $s: f^{-1} T(Y) \rightarrow T(X)$ is a locally Lipschitz splitting of the vector bundle sequence (4) - such a splitting always exists-we say that $s$ is bounded locally over $Y$ if for each $y_{0} \in Y$ there is a number $\eta_{0}>0$ and a neighborhood $V_{0}$ of $y_{0}$ such that $\|s(x)\|_{x} \leqq \eta_{0}$ for all $x \in f^{-1}\left(V_{0}\right)$; here the norm of $s(x)$ is that of $L(Y(f(x), X(x))$ induced from the norms $\alpha_{x}, \beta_{f(x)}$. We have the following theorem [28]:

Let $(X, \alpha),(Y, \beta)$ be Finsler $C^{1}$-manifolds, and suppose that $X$ is complete in the metric associated with $\alpha$. Let $f: X \rightarrow Y$ be a surjective $C^{1-}$ map whose differential $f_{*}(x)$ at every $x \in X$ is surjective and whose kernel is a direct summand of $X(x)$. If there is a locally Lipschitz splitting of (4) which is locally bounded over $Y$, then $f$ is a locally trivial fibration.

Local triviality means that every $y_{0} \in Y$ has a neighborhood $V$ for which $f^{-1}(V)$ is homeomorphic (preserving fibres) to $V \times f^{-1}\left(y_{0}\right)$. In a context in which $s$ is $C^{r}$ we can establish local $C^{r}$-triviality.

We now give an application taken from the theory of deformations of complex structures, based on fundamental work of $\mathrm{L}$. Ahlfors and L. Bers. 
EXAmple [28]. Let $U$ be the open upper half plane in the space $C$ of complex numbers, and $L^{\infty}(U, C)$ the Banach space of all bounded measurable complex functions on $U$. Let $D=\{z \in C:|z|<1\}$, endowed with the complete Riemannian structure of constant curvature $=-4$, and let $\sigma_{D}$ be its associated metric. Denote by $M=L^{\infty}(U, D)$ the open disc in $L^{\infty}(U, C)$; we view $M$ as a complex analytic infinite dimensional (nonseparable) manifold modeled on $L^{\infty}(U, C)$. Define the Finsler structure $\alpha$ on $M$ at the point $\mu \in M$ by

$$
\alpha_{\mu}(\nu)=\sup \left\{|\nu(z)|_{\mu(z)}: z \in U\right\}
$$

for all $\nu \in L^{\infty}(U, C)$, where $|\nu(z)|_{\mu(z)}$ denotes the Riemannian length of the vector $\nu(z)$ in the tangent space $D(\mu(z))$ to $D$ at $\mu(z)$. Then the metric

$$
\sigma\left(\mu_{0}, \mu_{1}\right)=\sup \left\{\sigma_{D}\left(\mu_{0}(z), \mu_{1}(z)\right): z \in U\right\}
$$

is that associated with $\alpha$, and $(M, \alpha)$ is complete.

A theorem of C. B. Morrey insures that for each $\mu \in M$ there is a unique solution $w^{\mu}$ of Beltrami's equation

$$
\frac{\partial w}{\partial \bar{z}}=\mu \frac{\partial w}{\partial z}
$$

in $C$, normalized by requiring that $0,1, \infty$ remain fixed and that $w^{\mu}$ be conformal in the lower half plane $U^{*}$. Let $B$ denote the complex (nonseparable) Banach space of holomorphic functions $\phi$ in $U^{*}$ whose norm

$$
|\phi|_{B}=\sup \left\{|z-\bar{z}|^{2}|\phi(z)|: z \in U^{*}\right\}<\infty .
$$

If $[w]=\left(w^{\prime \prime} / w^{\prime}\right)^{\prime}-\left(w^{\prime \prime} / w^{\prime}\right)^{2} / 2$ denotes the Schwarzian derivative (when defined), then the assignment $\mu \rightarrow\left[w^{\mu}\right]$ is a holomorphic map $\Phi: M \rightarrow B$ whose image $\Delta=\Phi(M)$ is a bounded open subset.

For any Fuchsian group $\Gamma$ (i.e., discrete group of Möbius transformations of $U$ ) let $M(\Gamma)$ denote the subspace of $\Gamma$-invariant elements of $M$, and $\Delta(\Gamma)=\Phi(M(\Gamma)) ; \Delta(\Gamma)$ is traditionally called the Teichmiiller space of $\Gamma$. The quotient Finsler structure on $\Delta(\Gamma)$ induced from that of $M$ has associated metric equivalent to that of Teichmüller's metric. The hypotheses of the preceding theorem are satisfied, whence $\Phi: M(\Gamma) \rightarrow \Delta(\Gamma)$ is a locally trivial fibration (with complex analytic fibres). In particular, whenever $\Delta(\Gamma)$ is contractible (and that is the case if $\operatorname{dim} \Delta(\Gamma)<\infty$ or if $\Gamma=\epsilon$ ), we conclude that for every $\phi \in \Delta(\Gamma)$ the fibre $\Phi^{-1}(\phi)$ is contractible.

6. Manifolds of maps. In this section we endow certain function spaces of maps (or sections of a fibre bundle, more generally) with 
differentiable manifold structures, necessarily of infinite dimension in general. The basic idea here is that a function space $\mathscr{F}(S, M)$ of maps of a space $S$ into a space $M$ of ten reflects much of the structure of $M$. In particular, if $M$ is a differentiable manifold, under certain conditions we have an induced manifold structure on $\mathscr{F}(S, M)$; the most important of these conditions are (1) that the topology on $\mathfrak{F}(S, M)$ be larger $(\geqq)$ than the uniform topology; (2) that $\mathfrak{F}$ behave well under maps of $S$ and $M$. We begin by illustrating these properties for special classes of maps of Banach spaces.

(A) Suppose that $E$ and $F$ are Banach spaces, and that $U$ is a subset of $E$.

Example 1. The totality of bounded maps $\phi: U \rightarrow F$ is a Banach space $B(U, F)$ with algebraic operations defined pointwise, and norm $|\phi|_{B(U, F)}=\sup \{|\phi(x)|: x \in U\}$; thus convergence of a sequence in $B(U, F)$ is precisely uniform convergence of that sequence on $U$. If $F$ is a Banach algebra, then so is $B(U, F)$. Any map $f: U_{1} \rightarrow U_{2}$ induces through composition a bounded linear map $\bar{f}: B\left(U_{2}, F\right) \rightarrow B\left(U_{1}, F\right)$. Dually, a map $g: F_{1} \rightarrow F_{2}$ carrying bounded sets of $F_{1}$ into bounded sets of $F_{2}$ induces a map $\bar{g}: B\left(U, F_{1}\right) \rightarrow B\left(U, F_{2}\right)$.

The vector subspace $B C(U, F)$ of bounded continuous maps is closed in $B(U, F)$; in general, $B C(U, F)$ is not separable. Furthermore, the evaluation map $B C(U, F) \times U \rightarrow F$ defined by $(\phi, x) \rightarrow \phi(x)$ is continuous. Note that if $U$ is compact and $V$ is open in $F$, then the subset $B C(U, V)=\{\phi \in B C(U, F): \phi(U) \subset V\}$ is an open submanifold. Ascoli's theorem gives the following characterization of the relatively compact sets $[23$, p. 137$]$.

Let $U$ be a compact subset of $E$. Then a subset $A$ of $B C(U, F)$ is relatively compact if and only if $A$ is equicontinuous, and for each $x \in U$ the set $A(x)$ of all $\phi(x)$ such that $\phi \in A$ is relatively compact in $F$.

Example 2. Now suppose that $U$ is open in $E$. Let $B C^{r}(U, F)$ denote the Banach space of $C^{r}$-maps $\phi: U \rightarrow F$ all of whose differentials $d^{k} \phi(0 \leqq k \leqq r<\infty)$ are bounded on $U$, with norm

$$
|\phi|_{r}=|\phi|_{B C^{r}(U, F)}=\sup \left\{\sum_{k=0}^{r}\left|d^{k} \phi(x)\right|: x \in U\right\} .
$$

Thus $B C^{0}(U, F)=B C(U, F)$. If $U$ is an open disc in $E$, then that norm is equivalent to

$$
\sup \left\{|\phi(x)|+\left|d^{r} \phi(x)\right|: x \in U\right\} .
$$

The space $B C^{\infty}(U, F)=\bigcap_{r=0}^{\infty} B C^{r}(U, F)$ is a Fréchet space with metric 


$$
\rho(\phi, \psi)=\sum_{r=0}^{\infty} \frac{|\phi-\psi|_{r}}{2^{r}\left[1+|\phi-\psi|_{r}\right]} .
$$

The map which assigns to each $\phi \in B C^{r}(U, F)$ its $k$ th differential is a bounded linear map $d^{k}: B C^{r}(U, F) \rightarrow B C^{r-k}\left(U, S L^{k}(E, F)\right)$ $(0 \leqq k \leqq r)$. If $f \in B C^{r}\left(U_{1}, U_{2}\right)$, where $U_{1}$ and $U_{2}$ are open subsets of $E$, then $f$ induces a bounded linear map $\bar{f}: B C^{r}\left(U_{2}, F\right) \rightarrow B C^{r}\left(U_{1}, F\right)$. Dually, we have the theorem [23, pp. 182-183, 2]:

Let $U$ be a compact differentiable n-manifold with boundary in the $n$-dimensional space $E$, so that $B C^{r}(U, F)=C^{r}(U, F)$, the Banach space of all $C^{r}$-maps of $U$ into $F$. Let $G$ be a Banach space. If $V$ is open in $F$ and $g \in B C^{r+s}(V, G)$, then the induced map $\bar{g}: B C^{r}(U, V) \rightarrow B C^{r}(U, G)$ is of class $C^{s}$ for all $0 \leqq s \leqq r$. Again, $B C^{r}(U, V)$ is open in $B C^{r}(U, F)$. If $U$ is open in $E$ and $\operatorname{dim} F<\infty$, then for any $r \geqq 1$ the inclusion map $B C^{r}(U, F) \rightarrow B C^{r-1}(U, F)$ is compact.

(B) Suppose that $M$ is a separable $C^{\infty}$-manifold modeled on a separable Hilbert space $E$, with tangent vector bundle $\pi: T(M) \rightarrow M$. If $M$ has a complete Riemannian structure and $m \in M$, we let $\exp _{m}: M(m) \rightarrow M$ denote the exponential map as in $\$ 5 \mathrm{E}$; its associated metric is denoted by $\rho=\rho_{M}$.

Let $S$ be a compact topological space, and $C=C(S, M)$ the totality of continuous maps $x: S \rightarrow M$; we give $C$ the topology of uniform convergence (in this case, the compact-open topology). The metric $\rho_{M}$ on $M$ induces an admissible metric on $C$ by

$$
\rho(x, y)=\sup \left\{\rho_{M}(x(s), y(s)): s \in S\right\} .
$$

$C$ is separable and complete, because $M$ is. The evaluation map $C(S, M) \times S \rightarrow M$ is continuous.

The following result is fundamental, and is typical of the various modifications discussed in this section [29]:

For any compact space $S$ and separable $C^{\infty}$-manifold $M$ modeled on a Hilbert space, the function space $C(S, M)$ is a $C^{\infty}$-manifold modeled on a separable Banach space. If $M$ is imbedded as a closed submanifold of a Hilbert space $V$, then $C(S, M)$ is a closed $C^{\infty}$-submanifold of the Banach space $C(S, V)$. A Riemannian structure on $M$ induces a Finsler structure on $C(S, M)$.

The basic idea in the proof is to introduce some complete Riemannian structure on $M$, and then to proceed as follows: (1) Construct the tangent vector space $C(x)$ to the map $x \in C(S, M)$ as the totality of continuous maps $u: S \rightarrow T(M)$ such that $\pi \circ u(s)=x(s)$ for all $s \in S$; then $C(x)$ is a Banach space with norm 


$$
|u|_{x}=\sup \left\{|u(s)|_{x(s)} ; s \in S\right\} .
$$

Note that $C(x)=C\left(x^{-1} T(M)\right)$, the space of continuous sections of the induced vector bundle $x^{-1} T(M) \rightarrow S$, through the map $x: S \rightarrow M$. (2) Use the exponential map of $M$ to construct an $x$-centered coordinate chart on $C(S, M)$; namely, since $x(S)$ is compact, there is a number $\lambda_{x}>0$ such that each point of $M$ whose distance from any $x(s)$ is less than $\lambda_{x}$ is joined to $x(s)$ by a unique geodesic arc of length $<\lambda_{x}$; then the map $s \rightarrow\left(\psi(u) s=\exp _{x(s)} u(s)\right.$ gives a bijective correspondence $\psi$ between the $\lambda_{x}$-disc of $C(x)$ centered at 0 and the $\lambda_{x}$-disc of $C(S, M)$ centered at $x$; whence a coordinate chart on $C(S, M)$. (3) Use the differentiability of $\exp : T(M) \rightarrow M$ to prove that the transitions between these charts are $C^{\infty}$-differentiable.

The differentiable structure of $C(S, M)$ is independent of the choice of the Riemannian structure on $M$, since $a C^{\infty}$-map $g: M_{1} \rightarrow M_{2}$ induces a $C^{\infty}$-map $\bar{g}: C\left(S, M_{1}\right) \rightarrow C\left(S, M_{2}\right)$. Furthermore if $g$ is an imbedding, then so is $\bar{g}$. If $f: S_{1} \rightarrow S_{2}$ is a continuous map of compact spaces, then $f$ induces a $C^{\infty}$-map $\bar{f}: C\left(S_{2}, M\right) \rightarrow C\left(S_{1}, M\right)$. If $f$ is an inclusion map and $\operatorname{dim} M<\infty$, then $\bar{f}$ is a $C^{0}$-locally trivial fibration over $\bar{f} C\left(S_{2}, M\right)$. (This last assertion is a slight modification of a theorem of $\mathrm{K}$. Borsuk.)

There are many variations of this example; beyond those described in the following sections, we mention three: (1) If $S_{0}$ is a closed subset of $S$ and $M_{0}$ a closed submanifold of $M$, then $C\left(S, S_{0} ; M, M_{0}\right)$ $=\left\{x \in C(S, M): x\left(S_{0}\right) \subset M_{0}\right\}$ is a closed submanifold of $C(S, M)$. (2) If $M$ is a $C^{s+2}$-manifold modeled on a $C^{s+2}$-smooth Banach space, then $C(S, M)$ has a $C^{s}$-manifold structure [2]. The point is that we can use a $C^{s+2}$-partition of unity to construct a $C^{s}$-spray [64] on $M$, which amounts to an exponential map exp: $T(M) \rightarrow M$ having the properties required for the above construction. (3) We can view $C(S, M)$ as the space of all continuous sections of the trivial bundle $S \times M \rightarrow S$, and then generalize the theorem, replacing $S \times M$ by a nontrivial bundle with $C^{\infty}$-fibres.

(C) Let $S$ be a compact $C^{\infty}$-manifold, and $0 \leqq r<\infty$; form the space $C^{r}(S, M)$ of $C^{r}$-maps $x: S \rightarrow M$ with the topology of uniform convergence of all derivatives of orders $\leqq r . C^{\infty}(S, M)$ is dense in every $C^{r}(S, M)$. If $M$ is imbedded as a closed submanifold of a Hilbert space $V$, then $C^{r}(S, M)$ is a closed subset of the Banach space $C^{r}(S, V)$ with norm as in (8).

For every $0 \leqq r<\infty$ the space $C^{r}(S, M)$ is a $C^{\infty}$-manifold modeled on a separable Banach space. A Riemannian structure induces a Finsler structure on $C^{r}(S, M)$. The same complementary remarks as in (B) apply here. In [24a] Douady has demonstrated an analogous theorem 
for $C^{s}(S, M)$ - suitably defined-where $S$ is a compact subset of a finite dimensional $C^{s}$-manifold, $M$ is an analytic manifold (real or complex), and $s=r+\alpha(0 \leqq \alpha<1$ and $r \geqq 1$ is an integer.

Furthermore, we have the following result [2]:

Suppose that both $S$ and $M$ are compact $C^{\infty}$-manifolds and that $N$ is a $C^{\infty}$-manifold modeled on a Hilbert space. Then the composition induces a map

$$
C^{r}(S, M) \times C^{r+s}(M, N) \rightarrow C^{r}(S, N)
$$

which is $C^{s}$.

Similarly, taking $S$ as a point we find that the evaluation map $C^{r}(M, N) \times M \rightarrow N$ is $C^{r}$. On the other hand, taking $S=M=N$, composition defines an associative continuous multiplication in the manifold $C^{r}(M, M)$ and a group structure in the $C^{r}$-diffeomorphisms of $M$, but the composition is not $C^{1}$ (pointed out by $\mathrm{R}$. Abraham).

(D) For applications [35], [36], [94] to potential theory and the calculus of variations it is important to work in the context of $L^{p_{-}}$ spaces, especially $p=2$. We now produce manifold structures on certain of these spaces.

Suppose that $S$ is a compact oriented Riemannian manifold of dimension $n$; we will write the volume $n$-form of $S$ as $* 1$. Fix a Hilbert space $V$. Then for any real number $p(1 \leqq p \leqq \infty)$ let $L_{0}^{p}(S, V)$ denote the Banach space of $L^{p}$-maps from $S$ to $V$ with norm (9) below with $r=0$. For any integer $r \geqq 0$ let $L_{r}^{p}(S, V)$ be the Banach space of such maps $x$, all of whose differentials $d^{k} x$ are of class $L^{p}(0 \leqq k \leqq r)$, whose norm can be given by

$$
|x|_{L_{r}^{p}(S, V)}=\left[\int_{S}\left\{\sum_{k=0}^{r}\left|d^{k} x(s)\right| 2\right\}^{p / 2} * 1\right]^{1 / p} .
$$

In particular, for all $r \geqq 0$ the space $L_{r}^{2}(S, V)$ is a Hilbert space with inner product

$$
\langle x, y\rangle_{L_{r}^{2}(S, V)}=\sum_{k=0}^{r} \int_{S}\left\langle d^{k} x(s), d^{k} y(s)\right\rangle * 1 .
$$

Clearly for $q \leqq p$ and $s \leqq r$ we have $L_{r}^{p}(S, V) \subset L_{r}^{q}(S, V)$ and $L_{r}^{p}(S, V) \subset L_{s}^{p}(S, V)$, since $S$ has finite volume. The following Sobolev imbedding theorems are basic [112]:

Let $1 \leqq p, q<\infty$. Then

(1) $L_{r}^{p}(S, V) \subset L_{s}^{q}(S, V)$ for $0 \leqq s \leqq r$ and $1 / q \geqq 1 / p-(r-s) / n \geqq 0$. 


$$
L_{r}^{p}(S, V) \subset C^{k}(S, V) \text { for } r>n / p+k .
$$

The inclusion sign indicates an algebraic subspace whose inclusion map is continuous. Furthermore, if $s<r$ and $1 / q>1 / p-(r-s) / n$, then both inclusion maps are compact. If $f: S_{1} \rightarrow S_{2}$ is a $C^{\infty}$-immersion, then $f$ induces a bounded linear map $\bar{f}: L_{r}^{p}\left(S_{2}, V\right) \rightarrow L_{r}^{p}\left(S_{1}, V\right)$ [91, Chapter XI D].

Let us take $k=0$ and $r>n / p$; then the topology on $L_{r}^{p}(S, V)$ is larger than the uniform topology. Furthermore, the composition of an $L_{r}^{p}$-map with a $C^{\infty}$-map is an $L_{r}^{p}$-map. Thus once again we are in a position to apply our construction to obtain the theorem [29], [35], [36], [89], [94]:

Let $M$ be a closed $C^{\infty}$-submanifold of a Hilbert space $V$, and let $L_{r}^{p}(S, M)=\left\{x \in L_{r}^{p}(S, V): x(s) \in M\right.$ for almost all $\left.s \in S\right\}$. Then $L_{r}^{p}(S, M)$ is closed in $L_{r}^{p}(S, V)$. If $r>n / p$, then $L_{r}^{p}(S, M)$ is a closed $C^{\infty}$-submanifold of $L_{r}^{p}(S, V)$. In particular, $L_{r}^{2}(S, M)$ is modeled on a Hilbert space, and the imbedding of $M$ in $V$ induces a complete Riemannian structure on $L_{r}^{2}(S, M)$.

If $g: M_{1} \rightarrow M_{2}$ is a $C^{\infty}$-map, then $g$ induces a $C^{\infty}$-map $\vec{g}: L_{r}^{p}\left(S, M_{1}\right)$ $\rightarrow L_{r}^{p}\left(S, M_{2}\right)$, if $r>n / p$.

Again, many variations of this example are possible: (1) We may permit $S$ to have a $C^{\infty}$-boundary, and take it into account in defining $L_{r}^{p}(S, M)$. (2) In the definition of $L_{r}^{p}(S, V)$ we can permit suitable weight factors (e.g., the Bessel potentials) for which the analogues of Sobolev's theorems are valid [51, Chapter 2]; in particular, we can define $L_{r}^{p}(S, V)$ for real numbers $r \geqq 0$.

(E) The inclusion maps $C^{r}(S, M) \rightarrow C^{0}(S, M) \quad(0 \leqq r \leqq \infty)$ and $L_{r}^{p}(S, M) \rightarrow C^{0}(S, M)(r>n / p)$ are homotopy equivalences. See [91, Chapter XI] for an elegant proof based on the lemma of PalaisSvarc. Thus the homotopy types of $C^{r}(S, M)$ and $L_{r}^{p}(S, M)$ depend only on the underlying topological structures of $S, M$.

7. Transversality of maps. This section describes uses of differentiable manifold structures in function spaces to establish generic properties of differentiable maps of differentiable manifolds. The results in (A) and (B) are due to Abraham [1], [2], extending fundamental work of R. Thom and H. Whitney (based on the BrownMorse-Sard theorem).

(A) Let $X, Y$ be $C^{r}$-manifolds $(r \geqq 1)$ modeled on Banach spaces. Consider a subset $a \subset C^{r}(X, Y)$ which is a $C^{r}$-manifold such that the evaluation map ev: $a \times X \rightarrow Y$ defined by $\operatorname{ev}(\phi, x)=\phi(x)$ is $C^{r}$. If $K$ is a subset of $X$ and $B$ a closed $C^{r}$-submanifold of $Y$, let $a_{K, B}$ 
$=\{\phi \in Q: \phi \mid K$ is transversal to $B\}$. The main theorem is the following:

If $K$ is compact, then $a_{K, B}$ is open in a. Let $\operatorname{dim} X=n<\infty$, codim $(Y, B)=q<\infty$, and $r>\max (n-q, 0)$. If the evaluation map is transversal to $B$ on $K$, then $a_{K, B}$ is residual in $a$ (i.e., is a countable intersection of open dense subsets).

That result can be modified in many different ways; for instance, (1) in the second assertion, instead of a closed submanifold $B$ in $Y$ we can take a $C^{r}$-map $\theta: B \rightarrow Y$ if both $B$ and $Y$ have finite dimension. If $r>\max (\operatorname{dim} X-\operatorname{dim} Y+\operatorname{dim} B, 0)$ and the evaluation map is transversal to $\theta$ on $K$, then $\mathfrak{Q}_{K, \theta}=\{\phi \in Q: \phi \mid K$ is transversal to $\theta\}$ is residual in $a$. (2) The theorems can be formulated for $C^{r}$-sections of a fibre bundle, generalizing the present case of sections of the product bundle $\mathrm{X} \times Y \rightarrow X$.

(B) The fact that $C^{r}(X, Y)$ is a $C^{r}$-manifold $(r<\infty)$ if $X$ is compact-and in particular is a Baire space, so that every residual set is dense-permits wide applicability of the preceding theorem. Let $\xi: V \rightarrow X$ be a $C^{r}$-fibre bundle, and $J^{k}(\xi): J^{k}(V) \rightarrow X$ its bundle of $k$ jets of sections $(0 \leqq k \leqq r)$; the fibre of $J^{k}(\xi)$ over $x \in X$ consists of the equivalence classes of sections $\phi$ defined in neighborhoods of $x$, identified when their differentials $d^{i} \phi(x)$ coincide $(0 \leqq i \leqq k)$. Its total space $J^{k}(V)$ is a $C^{r-k}$-manifold (and $J^{k}(\xi)$ is a vector bundle if $\xi$ is). The natural map $j^{k}: C^{r}(V) \rightarrow C^{r-k}\left(J^{k}(V)\right)$, which assigns to each $C^{r_{-}}$ section (assuming that one exists!) $\phi$ its equivalence class at each point $x \in X$, is called the $k$ th prolongation of $\phi ; j^{k}$ is an imbedding.

Now for simplicity of exposition take $V=X \times Y \rightarrow X$, so that $J^{k}(X \times Y)$ is identified with the bundle of $k$-jets of maps $X \rightarrow Y$. Then the above result produces the following theorem of Thom:

Suppose that $X$ and $Y$ are finite dimensional $C^{r}$-manifolds and $B$ a closed $C^{r-k}$-submanifold of $Y$. For every $F \in C^{r-k}\left(B, J^{k}(X \times Y)\right)$, the subset $\mathfrak{a}_{F}^{r}(X, Y)=\left\{\phi \in C^{r}(X, Y): j^{k}(\phi)\right.$ is transversal to $\left.F\right\}$ is residual in $C^{r}(X, Y)$, provided $r>\max \left\{\operatorname{dim} X-\operatorname{dim} J^{k}(X \times Y)+\operatorname{dim} B, 0\right\}$. In this assertion it is not necessary that $X$ be compact.

Applications of Thom's theorem include (1) Whitney's immersion theorems:

If $X$ and $Y$ are $C^{r}$-manifolds $(r \geqq 6)$ and $\operatorname{dim} Y \geqq 2 \operatorname{dim} X$, then the immersions are residual in $C^{r}(X, Y)$. If $n \geqq \max (\operatorname{dim} X, 2)$ and $\operatorname{dim} Y>2 \operatorname{dim} X$, then the imbeddings are residual in $C^{r}(X, Y)$.

(2) If $X$ is a $C^{r}$-manifold $(r \geqq 3)$ with $2 \leqq \operatorname{dim} X<\infty$, then the nondegenerate functions ( $\$ 9 \mathrm{~B}$ below) are residual in $C^{r}(X, R)$. 
(C) Let $X$ be a compact $C^{\infty}$-manifold and $A$ a closed submanifold of $X$. Then the inclusion map $f: A \rightarrow X$ induces a $C^{\infty}$-map $\bar{f}: C^{r}(X, Y)$ $\rightarrow C^{r}(A, Y)$ for any $0 \leqq r<\infty$; its differential $\bar{f}_{*}(\phi)$ at every point is surjective and has kernel a direct summand of the tangent space to $C^{r}(X, Y)$ at $\phi$. As in $\S 4 \mathrm{~B}$ we conclude that $\bar{f}$ is a split $C^{\infty}$-local fibration over its image; in particular, $\bar{f} C^{r}(X, Y)$ is a $C^{\infty}$-manifold, and for every $\phi \in \bar{f} C^{r}(X, Y)$ the subset $\bar{f}^{-1}(\phi)$ is a closed $C^{\infty}$-submanifold of $C^{r}(X, Y)$. The subsets $\operatorname{Im}^{r}(X, Y)$ and $\operatorname{Em}^{r}(X, Y)$ consisting of the immersions and imbeddings in $C^{r}(X, Y)$ are both submanifolds $(1 \leqq r<\infty)$; and the same properties of $\bar{f}$ pertain to these. The fibre space structure of $\bar{f}$ on $\operatorname{Im}^{r}(X, Y)$ and $\operatorname{Em}^{r}(X, Y)$ is basic in the immersion and imbedding theory of Hirsch-Smale and of J. Cerf.

8. Critical point theory.

(A) Let $\phi: X \rightarrow R$ be a differentiable function defined on a differentiable manifold $X$ modeled on a Banach space $E$. A critical point of $\phi$ is a point $x \in X$ at which the differential $\phi_{*}(x)=0$. A critical level of $\phi$ is a number $c \in R$ such that $\phi^{-1}(c)$ contains a critical point; from $\S 4 \mathrm{E}$ we see that if $c$ is not a critical level, then $\phi^{-1}(c)$ is a closed differentiable submanifold of $X$ of codimension 1 .

Suppose now that $E$ is a separable Hilbert space. Then $X$ admits a differentiable Riemannian structure $g$ as in $\$ 5 \mathrm{C}$, relative to which the gradient field $\nabla \phi \in C^{\infty}(T(X))$ of $\phi$ is characterized at each point $x$ as the contravariant representative of the differential:

$$
\phi_{*}(x) \cdot u=\langle\nabla \phi(x), u\rangle_{x} \text { for all } u \in X(x) .
$$

Of course $\nabla \phi$ depends very much on $g$, but its zeros do not, being just the zeros of $\phi *$.

We want next to decompose $X$ according to the lines of steepest descent of $\phi$; these are the trajectories of $-\nabla \phi$; i.e., the solutions of the differential equation

$$
\frac{d \gamma_{t}(x)}{d t}=-\nabla \phi\left(\gamma_{t}(x)\right) \text { with } \gamma_{0}(x)=x .
$$

Henceforth we will use the term trajectory to refer only to the positive half-solutions $\left(\gamma_{t}(x)\right)_{t \geq 0}$. As in the finite dimensional case we have the following elementary property:

Let $X$ be a complete Riemannian manifold and $\phi: X \rightarrow R(\geqq a) a$ differentiable function bounded below by $a$. Then the solutions of (10) are defined for all $t \in R$, and provide a full 1-parameter group of diffeomorphisms of $X$; its fixed points are just the critical points of $\phi$. 
(B) Let $X$ be a complete Riemannian manifold. We now establish conditions under which the function $\phi: X \rightarrow R(\geqq a)$ has sufficiently many critical points. In various forms-but always involving some sort of compactness-such conditions have been widely developed. (E.g., in the abstract variational theory of Morse [81], [82], the conditions of $\phi$-accessibility of $X$ and upper reducibility of $\phi$ suffice, in the presence of lower semicontinuity of $\phi$.)

The following hypothesis has been verified in several different concrete contexts [34], [35], [36]. It is the mildest tractable condition we know for proving the existence of critical points:

Condition (CT). There is a critical point of $\phi: X \rightarrow R(\geqq a)$ in the closure of every trajectory of $-\nabla \phi$.

This condition is assured whenever every trajectory is relatively compact; e.g., whenever $\nabla \phi$ maps trajectories in to relatively compact sets of $T(X)$.

Condition (CT) is satisfactory for basic existence theory, but is insufficient for a full-fledged Morse theory (see §9). The following much stronger hypothesis, formulated by Palais and Smale [94], [89], [110] and applied successfully by them, is satisfactory for that (whenever $\phi$ is nondegenerate in the sense of $\$ 9 \mathrm{~B}$ below). It is motivated by the facts that on any trajectory $\left(\gamma_{t}(x)\right)_{t \geq 0}$ we have $\phi$ bounded and

$$
\inf \left\{\left|\nabla \phi\left(\gamma_{t}(x)\right)\right|_{\gamma_{t}(x)}: t \in R(\geqq 0)\right\}=0 .
$$

Thus roughly speaking, Condition (CT) is Condition (C) along the trajectories of $\phi$.

Condition (C). Let $\phi: X \rightarrow R$ be a differentiable function. Whenever $\left(x_{i}\right)_{i \geq 1}$ is a sequence of points of $X$ on which $\phi$ is bounded and such that $\nabla \phi\left(x_{i}\right) \rightarrow 0$ (equivalently, $\phi_{*}\left(x_{i}\right) \rightarrow 0$ ), then there is a convergent subsequence.

A first result is the following:

If $X$ is a complete Riemannian manifold and $\phi: X \rightarrow R(\geqq a)$ satisfies Condition (CT), then there is a critical point in each component of $X$. If $\phi$ satisfies Condition (C), then there is a minimum point in each component.

Example. Suppose $\phi: X \rightarrow R$ is a proper function (i.e., the inverse image of any compact set of $R$ is compact in $X$ ). Then Condition (C) is satisfied; furthermore, $\operatorname{dim}(X)<\infty$, since $X$ is necessarily locally compact. 
ExAmple. Take for $X$ a Hilbert space and for $\phi: X \rightarrow R(\geqq 0)$ a continuous positive quadratic form. Then there is a unique bounded positive self-adjoint operator $v \in L(X)$ such that $\phi(x)=\langle v(x), x\rangle / 2$ for all $x \in X$, so that $v(x)$ is the gradient of $\phi$ at $x$. The trajectories of $\phi$ are given explicitly by $\gamma_{t}(x)=\exp (-v t) x$. As $t \rightarrow \infty$ each trajectory converges to a point in the kernel of $v$, the space of critical points of $\phi$. Thus Condition (CT) is satisfied; on the other hand, Condition (C) is not satisfied if the kernel of $v$ is not zero. (That these critical points appear as limits of the trajectories, rather than merely limit points, is due to the quadratic character of $\phi$.)

(C) We now give lower bounds-described in purely topological terms-for the number of critical points of a differentiable function $\phi: X \rightarrow \boldsymbol{R}$ satisfying Condition (C); the general idea here is due to Lusternik-Schnirelman, and has been fit to the present situation by J. T. Schwartz [107]. For simplicity we will suppose that $X$ is connected.

If $A$ is a closed subset of $X$, its category cat $(A)$ relative to $X$ is the least integer $k$ for which there exist $k$ closed (or open, since $X$ is an absolute neighborhood retract) sets $A_{1}, \cdots, A_{k}$ of $X$ whose union contains $A$ and such that each inclusion map $A_{j} \rightarrow X$ is null homotopic. If no such $k$ exists, we $\operatorname{define} \operatorname{cat}(A)=\infty ;$ then $\operatorname{cat}(A) \leqq \operatorname{dim}(A)$ +1 . If $m$ is an integer such that $1 \leqq m \leqq \operatorname{cat}(X)$, we define

$$
c_{m}(\phi)=\inf _{A \subset X}\{\sup (\phi(x): x \in A): \operatorname{cat}(A) \geqq m\} ;
$$

clearly $c_{1}(\phi) \leqq c_{2}(\phi) \leqq \ldots$. For any real number $c$ let $K_{c}$ be the set of critical points of $\phi$ in $X$ at the level $c$; Condition (C) implies that $K_{c}$ is compact. Then we have the following theorems [107]:

Let $X$ be a connected complete Riemannian manifold, and $\phi: X \rightarrow R$ a differentiable function satisfying Condition (C), not constant on any open subset of $X$. Suppose that $m \leqq n$ and that $-\infty<c_{m}(\phi)=c=c_{n}(\phi)$ $<+\infty$. Then $\operatorname{cat}\left(K_{c}\right) \geqq n-m+1$, and $\operatorname{dim}\left(K_{c}\right) \geqq n-m$. Thus even if $n=m, K_{c}$ is not empty. If $\phi$ is bounded below, then $\phi$ has at least cat $(X)$ critical points.

There is a well known lower bound for $\operatorname{cat}(X)$ : Define cuplong $(X)$ as the greatest integer $n$ such that for some coefficient field $F$ and elements $z_{i} \in H^{k_{i}}(X ; F)$ with $k_{i} \geqq 1(1 \leqq i \leqq n)$ we have the cup product $z_{1} \cdots z_{n} \neq 0$. Then $\operatorname{cat}(X) \geqq \operatorname{cuplong}(X)+1$.

These results have been extended by Palais [93] and F. Browder $[16$, in the case of manifolds modeled on uniformly convex Banach spaces] to complete Finsler manifolds, and to $C^{1}$-functions whose 
differentials satisfy a local Lipschitz condition and which satisfy Condition (C); that is important, for instance, for applications (as in $\S 10)$ to variational theory of a nonquadratic character. The idea is to introduce certain Lipschitz vector fields on $X$ with full trajectories, which play sufficiently well the role of the gradient field of $\phi$.

EXAMPLE. As in $\S 3 \mathrm{~B}$, let $B O(p)$ be the homogeneous space of $p$ planes through the origin of a separable infinite dimensional real Hilbert space $E$; then cuplong $(B O(p))=\infty$. In particular, the unit sphere $S$ of $E$ is the universal cover of $B O(1)$; and any differentiable function $\phi: S \rightarrow R(\geqq a)$ bounded below and even $(\phi(-x)=\phi(x)$ for all $x \in S$ ) which satisfies Condition (C) has infinitely many critical points on $S$. Applications of that property to show that certain eigenfunction problems have infinitely many solutions have been given (1) by Sobolev [60, Chapter VI, \$4] for Hammerstein integral equations, and (2) by F. Browder [16] for elliptic boundary value problems. See also Anosov [3] for the case of a function on $S$ which is invariant under certain finite cyclic groups of transformations on $S$.

(D) Suppose now that $X$ is a closed differentiable submanifold of a complete Riemannian manifold $Y$, and that $\phi: Y \rightarrow R$ is a differentiable function. Then for any $x \in X \subset Y$ we can form the gradient $\nabla_{Y} \phi(x) \in Y(x)$ relative to the Riemannian structure of $Y$, and the gradient $\nabla_{X} \phi(x) \in X(x)$ relative to the induced structure on $X$. Let $P_{x}: Y(x) \rightarrow X(x)$ be orthogonal projection, and $\nu(x)=\left(I-P_{x}\right) \nabla_{Y} \phi(x)$. Lagrange's method of multipliers is contained in the following assertion:

$\nabla_{X} \phi(x)=P_{x} \nabla_{Y} \phi(x)$; in particular, the point $x \in X$ is a critical point of $\phi: X \rightarrow R$ if and only if $\nabla_{Y} \phi(x)$ is orthogonal to $X(x)$ in $Y(x)$. Let $\left(\gamma_{t}\right)_{t \geq 0}$ be a trajectory of $\phi: X \rightarrow R$, and $\Gamma_{t}=i\left(\gamma_{t}\right)$ its image under the imbedding map $i: X \rightarrow Y$. Then

$$
\frac{d \gamma_{t}}{d t}=-\nabla_{X} \phi\left(\gamma_{t}\right) \text { when and only when } \frac{\partial \Gamma_{t}}{\partial t}+\nabla_{Y} \phi\left(\Gamma_{t}\right)=\nu\left(\Gamma_{t}\right) .
$$

The next typical criterion for establishing Conditions (CT) and (C) has been used in several different contexts, starting with the special case of Weyl's method of orthogonal projection [34-36; see also $\S \S 9,10$ below]:

Let $X$ be a closed submanifold of the Hilbert space $E$, and $\phi: E \rightarrow R$ a differentiable function. Suppose that

(1) the gradient $\nabla \phi=\nabla_{E} \phi$ has the form $\nabla \phi(x)=A(x)+K(x)$ for some compact map $K: X \rightarrow E$ and linear Fredholm map $A$ on $E$;

(2) if we decompose $\nabla \phi(x)$ into its tangential and normal components 


$$
\nabla \phi(x)=\nabla_{X} \phi(x)+\nu(x) \text { for all } x \in X,
$$

then $\nu: X \rightarrow E$ is compact on trajectories (resp., is a compact map);

(3) every subset of $X$ on which $\phi$ and $\left|\nabla_{X} \phi\right|$ are bounded is bounded in E. Then $\phi: X \rightarrow R$ satisfies Condition (CT) (resp., Condition (C)).

\section{Nondegenerate critical point theory.}

(A) The fundamental viewpoint of Morse theory [81], [82] of a real-valued function $\phi: X \rightarrow R$ on a topological space is that the order of the real number field induces a filtration $\left(\phi_{c}\right)_{c \in R}$ of $X$ by the sets $\phi_{c}=\{x \in X: \phi(x) \leqq c\}$. Then the relative homology groups $H\left(\phi_{b}, \phi_{a} ; F\right)$ with coefficients in a field $F$ can be used to define critical levels of $\phi$; and with mild restrictions on $\phi$ the filtration determines a homology spectral sequence which produces a form of the Morse inequalities (11) relating the number and types of critical levels to the homology of $X$.

In the special case that $\phi$ is a differentiable nondegenerate function on a compact manifold $X$, that theory admits a sequence of refinements, starting with Morse's inequalities as given below and culminating with Smale's handlebody theory. In the case that $X$ is an infinite dimensional manifold there are several special cases where Morse theory has been adapted and applied to concrete situations. (E.g., \$10D below, and Klingmann [59] for the study of a class of (possibly degenerate) differentiable functions on a Riemannian manifold which satisfy Condition (C) and for which a weak form of the Morse inequalities (11) is satisfied.) However, the most precise and satisfactory form of the theory at present (in both assertion and proof strictly analogous to that of the compact case) is that presented in (C) and (D) below.

(B) We say that a critical point $x_{0} \in X$ of $\phi$ is nondegenerate if $d^{2} \phi\left(x_{0}\right)$ (sometimes called the Hessian) is a nondegenerate bilinear form on $X\left(x_{0}\right)$; say that $\phi$ is nondegenerate if all its critical points are. The inverse function theorem implies that each nondegenerate critical point is isolated. The Morse index (resp., co-index) of $x_{0}$ [or of $\left.d^{2} \phi\left(x_{0}\right)\right]$ is the supremum of the dimensions of the linear subspaces of $X\left(x_{0}\right)$ on which $d^{2} \phi\left(x_{0}\right)$ is negative definite [resp., the index of $\left.-d^{2} \phi\left(x_{0}\right)\right]$.

Suppose that $x_{0}$ is a nondegenerate critical point of $\phi$ and that $\phi\left(x_{0}\right)=0$. An application of the spectral theorem to $d^{2} \phi\left(x_{0}\right)$ and Taylor's theorem shows that there is an $x_{0}$-centered chart $(\theta, U)$ in which $\phi\left(\theta^{-1}(x)\right)=|P x|^{2}-|(I-P) x|^{2}$ for all $x \in \theta(U)$, where $P$ is a continuous orthogonal projection in $E$. Thus the index (resp., co-index) of $x_{0}$ is the dimension of the range of $I-P$ (resp., the dimension of the 
range of $P$ ). This was established in the case $\operatorname{dim} E<\infty$ by M. Morse; his proof was modified by Rothe [99] in a special infinite dimensional case, and by Palais [89] to fit the present situation.

It is most important to be able to compute the index of a nondegenerate critical point. A good tool is provided by the Morse focal point theorem, which has been abstracted by Hestenes [48]: A resolution of the identity for a Hilbert space $E$ is a 1-parameter family $\left(E^{\lambda}\right)_{\lambda_{0} \leqq \lambda \leqq \lambda_{1}}$ of closed linear subspaces such that (1) $E^{\lambda_{0}}=0, E^{\lambda_{1}}=E$ : (2) if $\lambda \leqq \mu$, then $E^{\lambda} \subset E^{\mu}$; (3) if $\lambda_{0} \leqq \mu<\lambda_{1}$, then $E^{\mu}=\bigcap\left\{E^{\lambda}: \mu<\lambda \leqq \lambda_{1}\right\}$; (4) $E^{\mu}=E^{\mu-0}=$ Closure of $U\left\{E^{\lambda}: \lambda_{0} \leqq \lambda<\mu\right\}$ for every $\lambda_{0}<\mu \leqq \lambda_{1}$. Say that a quadratic form $\alpha \in S L^{2}(E, R)$ is a Legendre form if it is expressible as the difference $\alpha=\beta-\gamma$ of two quadratic forms, where $\beta$ is positive definite and $\gamma$ is weakly continuous. Given a Legendre form $\alpha: E \rightarrow R$, for each $\lambda_{0} \leqq \lambda<\lambda_{1}$ we choose a maximal linear subspace $C^{\lambda} C E^{\lambda}$ such that (1) no $x \neq 0$ in $C^{\lambda}$ is $\alpha$-orthogonal to any $E^{\mu}$ with $\mu>\lambda$; (2) if $x \in C^{\lambda}$, then $\alpha(x, y)=0$ for all $y \in E^{\lambda}$. Set $C^{\lambda_{1}}=0$. Then $c(\lambda)=\operatorname{dim} C^{\lambda}<\infty$, and only finitely many $c(\lambda) \neq 0$. If we let $i(\lambda)$ =index $(\alpha)$ on $E^{\lambda}$, then the focal point theorem can be stated as follows:

Let $\alpha: E \rightarrow R$ be a Legendre form, and $\left(E^{\lambda}\right)_{\lambda_{0} \leqq \lambda_{\S} \lambda_{1}}$ a resolution of the identity for $E$. Then for every $\lambda_{0} \leqq \lambda \leqq \lambda_{1}$ we have $c(\lambda)=i(\lambda+0)$ $-i(\lambda-0)$. Furthermore,

$$
\operatorname{index}(\alpha)=\sum_{\lambda_{0}<\lambda<\lambda_{1}} c(\lambda) .
$$

(C) Let $M$ be a differentiable manifold modeled on a Hilbert space and $N$ a closed submanifold; both $M$ and $N$ may have boundaries. We say that $M$ arises from $N$ by differentiably attaching a handle of type $(j, k)$ if (1) there is a homeomorphism $h$ of $D^{i} \times D^{k}$ (the product of $j$ - and $k$-dimensional closed discs $(0 \leqq j, k \leqq \infty))$ on to a closed subset of $M$, and $M=N \cup h\left(D^{j} \times D^{k}\right)$; (2) $h \mid S^{j-1} \times D^{k}$ is a diffeomorphism on to $\partial N \cap h\left(D^{i} \times D^{k}\right)$; (3) $h \mid \operatorname{Int}\left(D^{j}\right) \times D^{k}$ is a diffeomorphism onto $M-N$. The main theorem of differentiable Morse theory is the following [94], [89], [110]:

Let $X$ be a complete Riemannian manifold and $\phi: X \rightarrow R$ a differentiable nondegenerate function satisfying Condition (C). Then

(1) the critical levels of $\phi$ are isolated, and there are only a finite number of critical points of $\phi$ on any level;

(2) if there are no critical levels of $\phi$ in $[a, b]$, then $\phi_{a}$ is diffeomorphic to $\phi_{b}$;

(3) if $a<c<b$ and $c$ is the only critical level of $\phi$ in $[a, b]$ and $x_{1}, \cdots, x_{r}$ are the critical points of $\phi$ at level $c$, then $\phi_{b}$ is diffeomorphic 
to $\phi_{a}$ with $r$ handles of type $\left(j_{1}, k_{1}\right), \cdots,\left(j_{r}, k_{r}\right)$ disjointly and differentiably attached, where $j_{i}$ and $k_{i}$ are the index and co-index of $x_{i}$ $(1 \leqq i \leqq r)$.

We remark that in virtue of Dugundji's theorem [25] the sphere in infinite dimensional Hilbert space is a strong deformation retract of the disc, whence the homotopy type of $\phi_{b}$ in the third statement above is not altered by neglecting to attach any handle of infinite index.

An algebraic consequence of that structure theorem is contained in the Morse inequalities (whose proof can also be given on the basis of much less precise structure):

(4) If $a<b$ are not critical values of $\phi, \mu_{i}(a, b)$ the number of critical points of $\phi$ of index $i$ in $\phi^{-1}[a, b]$, and $\beta_{i}\left(\phi_{b}, \phi_{a} ; F\right)=\operatorname{dim} H_{i}\left(\phi_{b}, \phi_{a} ; F\right)$ for some coefficient field $F$, then for every integer $k \geqq 0$ we have

$$
\sum_{i=0}^{k}(-1)^{k-i} \beta_{i}\left(\phi_{b}, \phi_{a} ; F\right) \leqq \sum_{i=0}^{k}(-1)^{k-i} \mu_{i}(a, b) .
$$

Equality holds for $k$ large.

(5) If $\phi$ is bounded below, then for every $i$ we have $\beta_{i}(X ; F) \leqq \mu_{i}(\phi)$, the number of critical points of $\phi$ on $X$ of index $i$. Of course, these numbers may be infinite. If each $\mu_{i}(\phi)<\infty(0 \leqq i \leqq k)$, then

$$
\sum_{i=0}^{k}(-1)^{k-i} \beta_{i}(X ; F) \leqq \sum_{i=0}^{k}(-1)^{k-i} \mu_{i}(\phi) .
$$

A major qualitative aspect of these inequalities is that the left members involve only the homology structure of $X$, whereas the right members depend on the local analytic structure of $\phi$ (that latter being determined (as in Section 9B) in the neighborhood of a nondegenerate critical point).

EXAMPLE [75]. Let $X$ be a complete Riemannian manifold and $\phi: X \rightarrow R$ a nondegenerate function satisfying Condition (C). If $\phi$ is bounded below and has precisely one critical point, then $X$ is diffeomorphic to a Hilbert space. If $\phi$ is bounded and has just two critical points, then $X$ is homeomorphic to a sphere.

(D) There is a generalization [75], [76], [120] of nondegenerate Morse theory (made first in the compact case by $\mathrm{R}$. Bott) which is important for applications in infinite dimensions: Again, let $\phi: X \rightarrow R$ be a differentiable function on a complete Riemannian manifold. A connected closed submanifold $N$ of $X$ is a nondegenerate critical manifold of $\phi$ if every $x \in N$ is a critical point of $\phi$ whose tangent space $N(x) \subset X(x)$ coincides with the null space of the Hessian of $\phi$ 
at $x$; in particular, the restriction of the Hessian to the orthogonal complement $N^{\perp}(x)$ of $N(x)$ is nondegenerate. The index of $N$ is the constant value of the index of the Hessian on $N$. We define "differentiably attaching handlebundles" as we did "differentiably attaching handles" above, using disc bundles in place of products of discs.

Let $X$ be a complete Riemannian manifold and $\phi: X \rightarrow R$ a differentiable function satisfying Condition (C), whose critical set is a union of nondegenerate critical manifolds without interior. Then

(1) for any $a<b$ the critical set in $\phi^{-1}[a, b]$ is the union of a finite number of disjoint compact critical submanifolds of $\phi$;

(2) if there are no critical values of $\phi$ in $[a, b]$, then $\phi_{a}$ is diffeomorphic to $\phi_{b}$;

(3) if $a<c<b$ and $c$ is the only critical value of $\phi$ in $[a, b]$ and $\left(N_{j}\right)_{1 \unlhd j \leqq r}$ are the critical manifolds of $\phi$ at level $c$, then $\phi_{b}$ is diffeomorphic to $\phi_{a}$ with $r$ handlebundles disjointly and differentiably attached.

(4) If $a<b$ are not critical values of $\phi$ and $\left(N_{j}\right)_{1 \leq j \leq r}$ are the critical manifolds of finite indices $\left(k_{j}\right)_{1 \leq j \leq r}$ whose levels are in $[a, b]$, then for every integer $k \geqq 0$ we have

$$
\sum_{i=0}^{k}(-1)^{k-i} \beta_{i}\left(\phi_{b}, \phi_{a} ; Z_{2}\right) \leqq \sum_{j=0}^{r} \sum_{i=0}^{k}(-1)^{k-i} \beta_{i-k_{j}}\left(N_{j} ; Z_{2}\right) .
$$

Equality holds for $k$ large.

The choice of $\boldsymbol{Z}_{2}$ as coefficient field is dictated here by the unknown homological position of the $N_{j}$ in $X$.

10. Applications to variational theory.

(A) We offer first some illustrations of the use of differentiability in classical existence theory in the calculus of variations, followingin oversimplified form - the viewpoint developed by L. Tonelli and C. B. Morrey (see [79, Chapter I, II] and the references therein for the appropriate generalizations).

Let $\phi: U \rightarrow R$ be a $C^{2}$-function on a convex open subset $U$ of a Hilbert space $E$. First of all, if $\phi$ has a relative minimum at $x_{0} \in U$, then clearly $\phi_{*}\left(x_{0}\right)=0$. Secondly, it is an easy consequence of Taylor's theorem that if $d^{2} \phi(x ; v, v) \geqq 0$ for all $x \in U$ and $v \in E$, then $\phi$ is weakly lower semicontinuous in $U$; i.e., given any net $\left(x_{\alpha}\right)$ and $x_{0} \in U$ such that the inner products $\left\langle x_{\alpha}, y\right\rangle \rightarrow\left\langle x_{0}, y\right\rangle$ for all $y \in E$, then $\phi\left(x_{0}\right)$ $\leqq \lim$ inf $\phi\left(x_{\alpha}\right)$. It follows that such a $\phi$ assumes its absolute minimum on any weakly compact subset of $U$; but these are precisely the subsets which are weakly closed and bounded in norm [26, Chapter V].

EXAMPLE. If $a_{0}, \cdots, a_{n}$ are nonnegative real numbers, then the 
function $x \rightarrow \phi(x)=\sum_{k=0}^{n} a_{k}|x|^{k}$ is weakly lower semicontinuous on $E$; and $\phi$ assumes its minimum on any convex closed (in the norm topology of $E$ ) bounded subset of $E$.

ExAmple. Let $\alpha: E \rightarrow R$ be a Legendre form; that is equivalent to saying that $\alpha$ is a quadratic form on the Hilbert space $E$, expressible in the form $\alpha(x)=|A x|^{2} / 2$ for some left Fredholm operator $A$ [49]. Every Legendre form is weakly lower semicontinuous. This example abstracts the Dirichlet principle for elliptic operators, as we see from the following application to differential geometry [33]:

Let $M$ be a compact oriented differentiable manifold without boundary (for simplicity of exposition). Let $\xi, \eta$ be finite dimensional differentiable vector bundles over $M$, and $A$ an elliptic linear operator from the space of differentiable sections of $\xi$ to that of $\eta$. Given a differentiable section $\psi$ of $\eta$, there is a differentiable section $\phi$ of $\xi$ such that $A \phi=\psi$ if and only if $\psi$ is $L^{2}$-orthogonal to the kernel of the adjoint $A^{*}$ of $A$. Furthermore, the kernels of both $A$ and $A^{*}$ are finite dimensional.

To place this problem in the setting of Legendre forms, we proceed as follows: We introduce Riemannian structures on $M, \xi$, and $\eta$, relative to which we can construct the Hilbert spaces $L_{r}^{2}(\xi)$ of sections of $\xi$, as in $\S 6 \mathrm{D}$. If $A$ has order $r$, then $A: L_{r}^{2}(\xi) \rightarrow L_{0}^{2}(\eta)$ is a left Fredholm operator (this amounts essentially to Gårding's inequality for the strongly elliptic operator $\left.A^{*} A[33]\right)$. Therefore, the Dirichlet integral $\alpha(\phi)=|A \phi|_{0}^{2} / 2$ is weakly lower semicontinuous on $L_{r}^{2}(\xi)$. That was the viewpoint adopted in the proof of Hodge's theorem given in [80]; see also [33] for a simplified proof in the general case.

(B) The following general viewpoint of the calculus of variations in its differentiable context is that of [35], [36] and [94].

Let $M$ be a compact oriented Riemannian manifold, and $\gamma: V \rightarrow M$ a differentiable fibre bundle. A variational density on $\gamma$ of order $r$ is a function $f: J^{r}(V) \rightarrow R$ on the bundle of $r$-jets of sections of $\gamma$. We define the function $F: C^{\infty}(V) \rightarrow R$ through the diagram

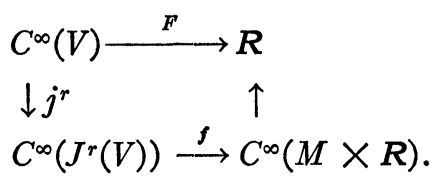

Here the right hand arrow denotes integration over $M$ of a realvalued function:

$$
F(\phi)=\int_{M} f\left(j^{r} \phi\right) * 1
$$


We say that $f$ is (quadratically) $C^{k}$-extensive if $F$ admits an extension to a $C^{k}$-function $L_{r}^{2}(V) \rightarrow R$. In the case that $\gamma$ is a vector bundle Smale [110] has given conditions to insure that $f$ be $C^{1}$-extensive, and that the second differential of the extended map have satisfactory properties. Of course, if $f$ is a quadratic form on a vector bundle $\xi$, then its restriction to any closed differentiable subbundle $\gamma$ is $C^{\infty}$ extensive.

The next theorem summarizes several recent contributions [35], [36], [94]. We state it for the case that the base manifold $M$ has no boundary; the modification of our formulation and methods to include boundary value problems, while presenting analytic and geometric features of interest, does not present severe difficulties.

Let $\gamma: V \rightarrow M$ be a differentiable fibre bundle over a compact oriented Riemannian manifold. Suppose that $f$ is a $C^{2}$-extensive, positive $(f \geqq 0)$, variational density on $\gamma$ of order $r>\operatorname{dim}(M) / 2$. Thus $f$ determines a $C^{2-}$ function $F: L_{r}^{2}(V) \rightarrow R(\geqq 0)$; and if $F$ satisfies Condition (CT), then $F$ has a critical point in every component of $L_{r}^{2}(V)$. If $F$ is nondegenerate and satisfies Condition (C), then we have a full Morse theory for $F$.

This result-and certain variations on it-provide existence theory in $L_{r}^{2}(V)$; taken together with certain examples [34], [35], it suggests that in general it is easier to solve high order variational problems than low order ones. Of course, there remains the local matter of showing that such $L_{r}^{2}(V)$-solutions have sufficiently good differentiability properties; see [79].

(C) EXAMpLE. Let $N$ be a compact Riemannian submanifold of the Euclidean space $R^{q}$; we choose an imbedding here solely for simplicity of exposition. We treat $M \times N$ as a trivial subbundle of the Riemannian vector bundle $M \times R^{q}$, and view its sections as maps from $M$ to $N$. For any $\phi \in L_{r}^{2}\left(M \times R^{q}\right)$ we define its $r$ th energy

$$
E_{r}(\phi)=\frac{1}{2} \int_{M}\left|\left(d+d^{*}\right)^{r} \phi\right|^{2} * 1,
$$

where $d^{*}$ denotes the adjoint of the exterior differentiation operator $d$, using the Riemannian structure of $M$. The restriction of $E_{r}$ to the closed submanifold $L_{r}^{2}(M \times N)$ is differentiable if $r>\operatorname{dim}(M) / 2$, and its extremals are called polyharmonic (or $r$-harmonic) maps of degree $r$ from $M$ to $N$. They are known to be differentiable.

If $M$ and $N$ are compact Riemannian manifolds, and $M$ is oriented, then every continuous map $\phi: M \rightarrow N$ is homotopic to an r-harmonic 
map, provided $r>\operatorname{dim}(M) / 2$. If $r=1$, or if $N$ has Riemannian sectional curvature Riem $(N) \leqq 0$, then the same conclusion holds.

This was proved a few years ago [34], [35], based on a form of the projection theorem of $\$ 8 \mathrm{D}$; a problem similar to the first assertion but involving boundary values was solved (with a similar projection method) by Palais [89]; his approach can be applied to produce that assertion with no essential difficulty.

Added in proof. A treatment has been given by J. S. Saber, Manifolds of maps, Ph.D. Thesis, Brandeis Univ., December, 1965.

ExAmple. If $\operatorname{dim} M=1$, then $M$ is diffeomorphic to the circle $S^{\mathbf{1}}$. In this case $L_{1}^{2}\left(S^{1}, R^{q}\right)$ is a Hilbert space with inner product

$$
\langle\phi, \psi\rangle_{I_{1}^{2}\left(S^{1}, R^{q}\right)}=\int_{S^{1}}\langle\phi(s), \psi(s)\rangle d s+\int_{S^{1}}\left\langle\phi^{\prime}(s), \psi^{\prime}(s)\right\rangle d s .
$$

The 1-harmonic maps $\phi: S^{1} \rightarrow N$ are just the closed geodesics of $N$ (parametrized proportionally to arc length). A treatment of geodesics and closed geodesics-in particular their Morse theory-from the present viewpoint has been given in [35], [58], [75], [89]. The existence of a nontrivial closed geodesic on $N$ (first proved by A. I. Fet) is an immediate consequence of the fact that $E_{1}$ satisfies Condition (C) ; furthermore, strong methods [58], [75] are available for establishing the existence of more closed geodesics (extending Morse's work on estimates of the number of closed geodesics on manifolds diffeomorphic to the $n$-sphere).

If $N$ is not compact, then Condition (C) is never satisfied; however, if $N$ is complete and its Riemannian structure possesses certain growth conditions at infinity, then Condition (CT) is satisfied [34] [36], [75].

(D) Applications of Morse theory have been made by $\mathrm{E}$. H. Rothe, in the case of certain nondegenerate functions $\phi$ defined on convex bounded open subsets $U$ of a Hilbert space $E$. (See [99], [100], and [101] for a survey. Also [102] for the theory under rather general boundary conditions.) He supposes that the gradient of $\phi$ is expressible in the form $x \rightarrow \nabla \phi(x)=x+K(x)$ for some compact map $K: U \rightarrow E$; then in the projection theorem of $\$ 8 \mathrm{D}$ conditions (1) and (2) are verified, whence some form of (3) insures that Condition (C) is satisfied. See also $\$ 11 \mathrm{~B}$ below.

11. Fixed point theory. Certainly among the most widely used algebraic topological tools in analysis are the theories of fixed points 
and topological degrees. We cannot begin to do justice to them in this report; we will treat their theoretical aspects, and refer to [21], $[60],[69],[70],[72]$ for applications to the existence, uniqueness, stability of solutions of ordinary and partial differential equations, and of integral equations.

(A) A first basic result is the Schauder-Tychonoff fixed point theorem [26, Chapter V]:

If $A$ is a convex compact subset of a locally convex topological vector space $E$ and $\Phi: A \rightarrow A$ a continuous map, then there is a point $a \in A$ for which $\Phi(a)=a$.

The main techniques of the proof involve the approximation of $\Phi$ by a map into a finite dimensional subspace of $E$, the application of fixed point theory in finite dimensions, and homotopy invariance properties. As a consequence we cite the following: If $A$ is a bounded, convex, closed subset of a reflexive Banach space $E$ (whence $A$ is weakly compact) and $\Phi: A \rightarrow A$ is weakly continuous, then $\Phi$ has a fixed point.

If $E$ is metrizable and $A$ a convex subset of $E$, then $A$ is compact if and only if $A$ has the fixed point property [57].

Important for other applications are the following properties:

(1) The closed convex envelope of a compact set (i.e., the intersection of all closed convex supersets of $A$ ) in a complete locally convex topological vector space $E$ is compact [13].

(2) If $A$ is a closed subset of a metrizable space $X$ and $\Phi: A \rightarrow E$ is a continuous map with relatively compact image, then there is a continuous extension of $\Phi$ to a map of $X$ into $E$ whose range lies in the closed convex envelope of $\Phi(A)$ [25].

In case $E$ is a Banach space the Schauder-Tychonoff theorem can be modified as follows [41], [98]:

If $\Phi: D \rightarrow E$ is a compact map of a closed disc $D$ of $E$ having $\Phi(\partial D) \subset D$, then $\Phi$ has a fixed point.

There are many variants of the Schauder-Tychonoff theorem (e.g., relaxing the condition of local convexity of $E$ (V. Klee), permitting set-valued mappings (S. Kakutani)), of which we cite the following two:

(1) Let $\Phi: E \rightarrow E$ be a compact map of the Banach space $E$, and $\lambda_{1} a$ real number $\left(0 \leqq \lambda_{1} \leqq 1\right)$. Then either there is a point $a \in E$ with $\lambda_{1} \Phi(a)$ $=a$, or $\left\{x \in E: \lambda \Phi(x)=x\right.$ for some $\left.\lambda\left(0<\lambda<\lambda_{1}\right)\right\}$ is unbounded [21, Chapter III].

(2) If $\Phi: E \rightarrow E$ is a compact map such that for some positive integer $m$ the iterate $\Phi^{m}(E)$ is bounded, then $\Phi$ has a fixed point [15], [71]. 
ExAmple. Some compactness assumption is essential in these assertions. If $D$ denotes the closed unit disc in a normed linear space $E$, then there are fixed point free maps $\Phi: D \rightarrow D$ if and only if $\operatorname{dim} E$ $=\infty$ [25]. If $E$ is a separable infinite dimensional Hilbert space, then Kakutani (see [56], [57] for generalizations) has exhibited a fixed point free homeomorphism of $D$ onto itself; in this order of ideas Klee [56] has shown that the sphere $\partial D$ is homeomorphic to any closed convex body (i.e., subset with an interior point) in $E$. Every infinite dimensional compact convex subset of a normed linear space is homeomorphic to the Hilbert parallelotope [57].

(B) We next obtain a count for the algebraic number of fixed points of certain maps; that is achieved through the Leray-Schauder degree (developed in [72] for Banach spaces, and extended in [70], [86] to locally convex spaces).

Let $U$ be an open subset of the locally convex topological vector space $E$. Suppose that $\phi: \vec{U} \rightarrow E$ is a continuous map expressible in the form $x \rightarrow \phi(x)=x-\Phi(x)$ for all $x \in \bar{U}$, where $\Phi(\bar{U})$ is relatively compact. (It might be helpful to think of $\phi$ as a vector field on $\bar{U}$.) Then for any point $b \in E-\phi($ bdy $U)$ the Leray-Schauder degree $d[\phi, U, b]$ of $\phi$ in $U$ relative to $b$ is an integer defined [70], [86], through a finite dimensional approximation of $\Phi(\bar{U})$, roughly speaking as the algebraic number of times that $b$ is covered by $\phi$-images of points of $U$. It has the following properties:

(1) If $d[\phi, U, b] \neq 0$, then $b \in \phi(U)$.

(2) If $U_{1}, U_{2}$ are disjoint open subsets of $U$ such that $\bar{U}=\bar{U}_{1} \cup \bar{U}_{2}$ and $b \in E-\phi\left(\right.$ bdy $U_{1} \cup$ bdy $\left.U_{2}\right)$, then

$$
d\left[\phi, U_{1} \cup U_{2}, b\right]=d\left[\phi, U_{1}, b\right]+d\left[\phi, U_{2}, b\right] .
$$

(3) The integer $d[\phi, U, b]$ is invariant under continuous deformations of $\phi, U, b$, if $\phi_{t}$ maintains its form $x \rightarrow x-\Phi_{t}(x)$ with the deformation $\Phi_{t}(\bar{U})$ contained in a compact set for $0 \leqq t \leqq 1$, and $b$ never lies in $\phi(\operatorname{bdy}(U))$.

(4) If $U_{0}$ is an open subset of $U$ containing $\phi^{-1}(b)$, then

$$
d\left[\phi, U_{0}, b\right]=d[\phi, U, b] .
$$

(5) Let $V$ be an open subset of $E$ containing $\phi(\bar{U})$, and $\left(V_{i}\right)$ the components of $V-\phi($ bdy $U)$. If $\psi: \bar{V} \rightarrow E$ is a continuous map of the form $x \rightarrow \psi(x)=x-\Psi(x)$ with $\Psi(\bar{V})$ relatively compact, and $b \in E$ $-\psi \circ \phi($ bdy $U)-\psi($ bdy $V), b_{i} \in B_{i}$, then

$$
d[\psi \circ \phi, U, b]=\sum_{i} d\left[\phi, U, b_{i}\right] d\left[\psi, V_{i}, b\right] .
$$


The Schauder-Tychonoff theorem is an easy consequence of these properties. One can prove the following restricted version of Brouwer's theorem on invariance of domain [70], [106], [86]:

If $E$ is a Fréchet space and $\phi: \bar{U} \rightarrow E$ is a one-one map of the form above, then $\phi(U)$ is open in $E$, and for any $b \in \phi(U)$ we have $d[\phi, U, b]$ $= \pm 1$. Schauder [105] has used such invariance of domain in Banach spaces in studying solutions (in particular, in establishing existence from uniqueness assumptions) of second order elliptic quasi-linear equations with Dirichlet data. Invariance of domain also implies the Fredholm alternative for linear operators $\phi$ such that $x \rightarrow x-\phi(x)$ is compact [69], [70].

(C) Let $U$ be a bounded open subset of a Banach space $E$, and $a \in U$ an isolated fixed point of $\Phi$ and $U_{a}$ a neighborhood of $a$ in $U$ containing no other fixed point of $\Phi$. Letting $\phi=I-\Phi$, then $d\left[\phi, U_{a}, 0\right]$ is independent of the choice of $U_{a}$, and is henceforth denoted by $d[\phi, a]$, the index of $a$. If $\phi^{-1}(0)$ consists of isolated points $a_{1}, \cdots, a_{s}$ $\in U$, then $d[\phi, U, 0]=\sum_{j=1}^{s} d\left[\phi, a_{j}\right]$ is called the algebraic number of zeros of $\phi$ in $U$. In particular, suppose that $\Phi: \vec{U} \rightarrow E$ is Fréchet differentiable at a fixed point $a \in U$. If +1 is not an eigenvalue of the differential $\Phi_{*}(a)$, then $a$ is an isolated zero of $\phi$ with index

$$
d[\phi, a]=(-1)^{\alpha},
$$

where $\alpha$ is the sum of the orders of the eigenvalues of the compact linear operator $\Phi_{*}(a)$ in the open interval $(0,1)[60],[72]$.

If $\phi$ is the gradient field $\nabla \psi$ of a nondegenerate function $\psi: U \rightarrow R$ defined on a bounded open convex subset of a separable Hilbert space, and $a$ is a critical point of $\psi$, then $\alpha$ is the Morse index of $\psi$ at $a$. Again letting $\mu_{i}(\psi)$ denote the $i$ th Morse number of critical points in $U$ of $\psi$, we obtain the formula of Rothe [99]:

$$
d[\nabla \psi, U l 0]=\sum_{i \geqq 0}(-1)^{i} \mu_{i}(\psi) .
$$

In particular, $|d[\nabla \psi, U, 0]|$ is a lower bound for the number of critical points of $\psi$ in $U$. See [100] for an extension to the case that $\psi$ is degenerate. This formula has been used by Rothe [99], [60] to establish existence and qualitative properties of certain systems of nonlinear integral equations, including the Hammerstein equation.

(D) The following result is a generalization and application of the Lefschetz fixed point theorem for absolute neighborhood retracts. We write $H(X ; Q)$ for the direct sum of the singular homology groups $H_{i}(X ; Q)$ of $X$ with rational coefficients; and for a map $\Phi: X \rightarrow X$ let $\Phi_{*}$ denote the direct sum of the induced endomorphisms $\Phi_{i}: H_{i}(X ; Q)$ $\rightarrow H_{i}(X ; Q)$. 
Let $X$ be a $C^{0}$-manifold modeled on a Fréchet space, and $\Phi: X \rightarrow X a$ continuous map with relatively compact image. Then the image $\Phi_{*} H(X ; Q)$ of the rational homology group is finite dimensional, so that the Lefschetz number

$$
\Lambda(\Phi)=\sum_{i \geq 0}(-1)^{i} \text { Trace }\left(\Phi_{i}\right)
$$

of $\Phi$ is defined. If $\Lambda(\Phi) \neq 0$, then $\Phi$ has a fixed point.

Suppose that $X$ is acyclic over $Q$ (i.e., $H_{i}(X ; Q)=0$ for all $i>0$, and $\left.H_{0}(X ; Q)=Q\right)$. Then $\Phi$ has a fixed point.

The idea here is that $X$ is imbeddable as a closed subset of a Banach space $V$ as a retract of a neighborhood $U$ in $V$. Then we can construct a compact absolute neighborhood retract $P$ such that $\Phi(X)$ $\subset P \subset U$, and a map $\Psi: P \rightarrow P$ with $\Lambda(\Psi)=\Lambda(\Phi)$ and such that any fixed point of $\Psi$ in $X$ is a fixed point of $\Phi$; but we can apply Lefschetz's theorem to $\Psi$. (A special case of this theorem ( $X$ is a $C^{3}$-manifold modeled on a $C^{3}$-smooth separable Banach space) has been found independently by F. Browder [17], and then generalized by him to iterated maps; see also [71].) As a simple illustration, let $X$ be an infinite dimensional projective space (real, complex, or quaternionic); then any continuous map $f: X \rightarrow X$ with relatively compact image has a fixed point.

If in the above theorem $\Phi$ has only isolated fixed points $a_{1}, \cdots, a_{s}$ $\in X$, then with each we can attach the index $d\left[\Phi, a_{j}\right]$, and

$$
\Lambda(\Phi)=\sum_{j=1}^{s} d\left[\begin{array}{ll}
\Phi & a_{j}
\end{array}\right] .
$$

See [71] for the relations between local degrees and the Lefschetz number. In the special case that $X$ is a Riemannian manifold, each $d\left[\Phi, a_{j}\right]$ can be computed analytically.

(E) Smale [111] has suggested the following notion (generalizing a concept developed extensively by L. Pontrjagin in the finite dimensional case) of degree for certain maps of differentiable manifolds. Let $X$ and $Y$ be connected separable $C^{r}$-manifolds modeled on Banach spaces (which we will suppose infinite dimensional), and $f: X \rightarrow Y$ a proper Fredholm $C^{r}$-map of index $(f)=p \geqq 0$ and $r>p+1$. Then for all points $b \in Y$ except for a meager subset of $Y$ the set $f^{-1}(b)$ is a closed $C^{r}$-submanifold of $X$ of dimension $p$, as in $\$ 4 \mathrm{E}$. That determines a definite element $\gamma(f)$ of the unoriented bordism group $\mathfrak{N}_{p}(X)$ of $X$, which is independent of the choice of $b$. (For definition and calculation of $\mathfrak{N}_{p}(X)$ see $[19, \S \S 4,8]$.) $\gamma(f)$ is called the degree mod 2 of $f$. That concept has many properties of degree; e.g., (1) if $f_{0}, f_{1}$ : 
$X \rightarrow Y$ are Fredholm homotopic, then $\gamma\left(f_{0}\right)=\gamma\left(f_{1}\right)$; (2) if $\gamma(f) \neq 0$, then $f$ is surjective. If index $(f)=0$, then $\gamma(f)$ can be interpreted as an integer modulo 2; and in case $X$ is an open subset of a Banach space $E=Y$, then $\gamma(f) \equiv d[f, U, b] \bmod 2$.

12. Homological duality. In this section we describe certain aspects of manifolds centering around Poincaré and Alexander-Pontrjagin duality. We begin with a theorem (closely related to the GysinThom isomorphism theorem) on the cohomology of a closed finite codimensional submanifold, which is a combination of these two dualities.

(A) Let $X$ be a $C^{0}$-manifold modeled on a locally convex topological vector space $E$, and $A$ a closed submanifold of codimension $p \geqq 1$. If to each open subset $U$ of $X$ we assign the relative singular cohomology group $H^{*}(U, U-A)$ with integer coefficients, and to each open $V \subset U$ the natural homomorphism $H^{*}(U, U-A) \rightarrow H^{*}(V, V-A)$, then that assignment determines a sheaf $J \rightarrow A$ (the orientation sheaf of the pair $(X, A))$ locally isomorphic to the integers. We will let $H^{*}(A ;$ J $)$ denote the singular cohomology of $A$ with local coefficients $J$.

The following theorem is the principal result [30], [32], [87]; a generalization can be given, taking into account supports, general coefficient sheaves, and multiplicative structure.

Let $X$ be a $C^{0}$-manifold modeled on a locally convex topological vector space, and $A$ a closed $p$-codimensional submanifold $(p \geqq 1)$. There is a canonical isomophism for all $i \in \boldsymbol{Z}$

$$
\phi: H^{i}(A ; \Im) \rightarrow H^{i+p}(X, X-A) .
$$

Both cohomology groups are based on singular cochains; the right member uses relative cohomology with integer coefficients. The proof has two aspects: (1) If $X$ is any paracompact space and $A$ any closed subset, then the graded sheaf $J \rightarrow A$ can be defined, and there is a spectral sequence with $E_{2}=\check{H}^{*}(A, \mathfrak{I})$, Čech cohomology with coefficients in the sheaf $\mathfrak{I}$, converging to $H^{*}(X, X-A)$; (2) a local study (admitting interesting generalizations [87]) of a neighborhood of $A$ to produce triviality of the spectral sequence.

We say that the pair $(X, A)$ is oriented if the sheaf $\exists \rightarrow A$ is simple, and if a definite isomorphism $J \approx Z$ has been chosen. The class $\phi(1)$ $=a \in H^{p}(X, X-A)$ is called the fundamental class of $(X, A)$, and we can express the isomorphism $\phi$ in the form $\phi(x)=x \cdot a$ (cup product). Thus we have the interpretation of $H^{*}(X, X-A)$ as a free $H^{*}(A)$ module of rank 1 , generated by the fundamental class $a$. Furthermore, we have an exact sequence 
(12) $\cdots \rightarrow H^{i-1}(X-A) \stackrel{\lambda}{\rightarrow} H^{i-p}(A) \stackrel{\eta}{\rightarrow} H^{i}(X) \stackrel{\xi^{*}}{\rightarrow} H^{i}(X-A) \rightarrow \cdots$

where $\xi: X-A \rightarrow X$ is the inclusion map, $\eta$ corresponds to the Gysin homomorphism (of the normal bundle of $A$ in $X$, when that makes sense), and $\lambda$ is the cohomology analogue of Alexander linking between cycles of $A$ and cycles of $X-A$.

If $(X, A)$ is an oriented pair we can define (following the procedure of Thom) its Euler class $W_{p}(X, A)=\phi^{-1}[\phi(1) \cdot \phi(1)] \in H^{p}(A)$. (In the differentiable case this is just the Euler class of the transverse bundle of $A$ in $X$.) More generally, for any $p$-codimensional submanifold $A$ in $X$ we can define the total Stiefel-Whitney class $w(X, A)$ $=\phi^{-1} \mathrm{Sq} \phi(1)$ using $Z_{2}$-coefficients, where Sq denotes the Steenrod square in cohomology over $\boldsymbol{Z}_{2}$.

EXAMPLE. If the inclusion $\alpha: A \rightarrow X$ imbeds $A$ as a deformation retract of $X$, then the homomorphism $\eta$ of the above exact sequence is given by multiplication: $\eta(x)=\left(\alpha^{*}\right)^{-1}\left[x \cdot W_{p}(X, A)\right]$. If $X$ is such a neighborhood of $A$ in a manifold $Y$, then $W_{p}(X, A)$ is an invariant of the imbedding $A \rightarrow Y$.

(B) The Brouwer-Jordan separation theorem is intimately connected with Alexander-Pontrjagin duality on manifolds:

Let $X$ be a $C^{0}$-manifold modeled on a locally convex topological vector space, and $A$ a closed submanifold of codimension 1. If $H^{1}(X)=0$ and $\beta_{0}(X)=\operatorname{dim} H_{0}(X)$ is the number of components of $X$, then

$$
\beta_{0}(X-A)=\beta_{0}(X)+\beta_{0}(A) \text {. }
$$

Similarly, (1) if $p \geqq 2$ and $X$ is connected, then so is $X-A$; (2) if $X$ is acyclic, then we have the isomorphism $\lambda: H^{i}(X-A) \rightarrow H^{i-p+1}(A)$ for all $i \geqq 1$. These properties follow from the exact sequence (12)in particular, from the segment (using $\boldsymbol{Z}_{2}$-coefficients throughout if $(X, A)$ is not oriented)

$$
0 \rightarrow H^{0}(X) \rightarrow H^{0}(X-A) \stackrel{\lambda}{\rightarrow} H^{1-p}(A) \rightarrow 0 .
$$

In another direction we have the following separation property [39], [70], closely related to the invariance of domain of $\$ 11 \mathrm{~B}$ :

Let $E$ be a Banach space, $A$ a closed bounded subset, and $\phi: A \rightarrow E$ a homeomorphism of $A$ onto a closed subset $\phi(A)$. If the map $x \rightarrow x-\phi(x)$ is compact, then $E-A$ and $E-\phi(A)$ have the same number of components.

On the other hand, it has been established by Klee [56; this reference contains several generalizations to Banach spaces of such theorems] that if $A$ is compact and $E$ is an infinite dimensional Hilbert 
space, then $E-A$ is homeomorphic to $E$. Bessaga [8] has shown that $E-0$ is $C^{\infty}$-diffeomorphic to $E$; furthermore, the diffeomorphism can be put in such a form that it produces a $C^{\infty}$-diffeomorphism of $E$ on to its unit sphere $S$.

(C) Closely related to Alexander-Pontrjagin duality in a Banach space is the following treatment of cohomotopy theory, due to $\mathrm{K}$. Gęba [39]. Fix an infinite dimensional Banach space $E$, and choose a decreasing sequence of closed linear subspaces $E=E^{\infty} \supset E^{\infty-1}$ $\supset E^{\infty-2} \supset \cdots$ such that $\operatorname{codim}\left(E^{\infty}, E^{\infty-k}\right)=k$ for all $k \geqq 0$; let $P^{\infty-k}=E^{\infty-k}-0$. For any closed bounded subset $X$ of $E$ we consider the continuous maps $\phi: X \rightarrow P^{\infty-k}$ of the form $\phi(x)=x-\Phi(x)$, where $\Phi: X \rightarrow E$ is compact. Say that two such maps $\phi, \psi: X \rightarrow P^{\infty-k}$ are specially homotopic if there is a homotopy $h$ between them of the form $h_{t}(x)=x-H_{t}(x)$, where $H: X \times I \rightarrow E$ is compact. These special homotopy classes form an abelian group $\pi^{\infty-k}(X)$, called the $k$ th cohomotopy group of $X$, with addition patterned after $K$. Borsuk's cohomotopy addition. (Similarly, if $C$ is a closed subset of a finite dimensional subspace of $E$ and $[X, E-C]_{S}$ denotes the totality of special homotopy classes of maps $\phi: X \rightarrow E-C$, then $[X, E-C]_{S}$ has a natural abelian group structure $[7,39 \mathrm{a}]$.) That definition can be relativized to produce an abelian group $\pi^{\infty-k}(X, A)$ for pairs $(X, A)$, where $A$ is a closed subset of $X$; if $f:(X, A) \rightarrow(Y, B)$ is a map of pairs of the form $f(x)=x-F(x)$, where $F$ is compact, then $f$ induces a homomorphism $f^{\infty-k}: \pi^{\infty-k}(Y, B) \rightarrow \pi^{\infty-k}(X, A)$ for all $k \geqq 0$. Gegba [38], [39] has shown that $\left(\pi^{\infty-k}, f^{\infty-k}\right)$ forms an extraordinary cohomology theory (in the category of spaces, maps, and homotopies under consideration). Furthermore, Spanier-Whitehead duality is satisfied [39]:

If $X$ is a closed bounded subset of a Banach space E, then there is a canonical isomorphism $\pi^{\infty-k}(X) \rightarrow \sum_{k}(E-X)$ for all $k \geqq 0$, where $\sum_{k}(E-X)$ are the $S$-homotopy groups [113], defined as the direct limit of the sequence

$$
\pi_{k}(E-X) \rightarrow \pi_{k+1}(S(E-X)) \rightarrow \cdots \rightarrow \pi_{k+j}\left(S^{j}(E-X)\right) \rightarrow \cdots,
$$

where $S^{i}(E-X)$ denotes the jth iterated suspension of the space $E-X$.

In particular, $\pi^{\infty}(X)$ is a free abelian group, and there is a bijective correspondence between its generators and the bounded components of $E-X$.

If we take $X=S$, the unit sphere in a Hilbert space $E$, then $\pi^{\infty-k}(S)$ is identified with the $k$-stem of the homotopy groups of spheres: 


$$
\pi^{\infty-k}(S) \approx \lim _{r \rightarrow \infty} \pi_{r+k}\left(S^{r}\right)
$$

On the other hand, $\pi^{\infty-k}(S)$ can be identified with special homotopy classes of maps $\phi: S \rightarrow P^{\infty}$ of the form $\phi(x)=A(x)-\Phi(x)$, where $A$ is a fixed linear Fredholm operator on $E$ of index $k$, and $\Phi: S \rightarrow E$ is a compact map [38], [39], [115]. The special case $k=0$ is due to Rothe [98], as an application of the Leray-Schauder degree; it can be viewed as a variant of a classification theorem of $\mathrm{H}$. Hopf.

(D) Alexander-Pontrjagin duality suggests that a $p$-codimensional submanifold $A$ of $X$ should represent some sort of homology class of $X$ of dimension $\infty-p$; and from intersection theory of such submanifolds there should be a form of Poincaré duality relating singular p-dimensional cohomology groups $H^{p}(X)$ to $p$-codimensional homology groups $\mathfrak{H C}_{\infty-p}(X)$. We are not now in possession of a definitive theorem of that kind; however, the following construction may be of temporary interest [7]:

Let $U$ be an open subset of a separable Hilbert space $V$. Choose an orthonormal base $\left(e_{i}\right)_{i \geqq 1}$ for $V$, and let $V_{k}$ be the space spanned by $\left(e_{i}\right)_{1 \leqq i \leqq k}$; set $U_{k}=U \cap V_{k}$. Then the natural map inj $\lim U_{k} \rightarrow U$ is a homotopy equivalence by the lemma of Palais-Švarc, so that we have the canonical isomorphism $H^{i}(U)=H^{i}\left(\operatorname{inj} \lim U_{k}\right)$ for all $i \in Z$. On the other hand, each $U_{k}$ is a $k$-dimensional oriented manifold, so that Poincaré duality in finite dimensions defines an isomorphism

$$
D_{k}: H^{i}\left(U_{k}\right) \rightarrow L F H_{k-i}\left(U_{k}\right),
$$

where the right member denotes the singular $(k-i)$-homology group of $U_{k}$ based on locally finite chains. For any coefficient group $G$ and any $i \in Z$ we let $\mathfrak{H}_{\infty-i}(U ; G)$ denote the inverse limit

$$
\mathfrak{H}_{\infty-i}(U, G)=\operatorname{proj} \lim L F H_{k-i}\left(U_{k} ; G\right)
$$

determined by the inclusions $\eta: U_{k} \rightarrow U_{k+1}$ and the duality isomorphisms $\mathscr{D}_{k}$. The elements of $\mathfrak{T C}_{\infty-i}(U ; G)$ can be viewed as sequences of cycles $\left(\cdots, z_{k-i}, z_{k+1-i}, \cdots\right)$, where $z_{k-i}=\eta_{\text {ด }}\left(z_{k+1-i}\right)$ and $\eta_{\text {ด }}$ is the Umkehrungshomomorphismus of the inclusion map $\eta$.

Let $U$ be an open subset of a separable infinite dimensional Hilbert space, and $G$ a coefficient group. Assume either (1) $H_{i}(U)$ is finitely generated for all $i$, or (2) $G$ is a field. Then we have the canonical isomorphism

$$
\text { D: } H^{i}(U ; G) \rightarrow \mathfrak{H C}_{\infty-i}(U ; G)
$$

for all $i \in \boldsymbol{Z}$. 
Examples [7] show that some restrictions such as (1) or (2) are necessary; however, we do not know whether that is merely due to a defect in our definition of $\mathfrak{H}_{\infty-i}(U ; G)$.

(E) The following constructions were first given in [30], [32] in a differentiable context (using the inverse function theorem), and in the present more general and improved form in Namioka [87], using a local fibre structure of certain maps to replace the inverse function theorem.

Let $M$ be a manifold modeled on a locally convex topological vector space, $S$ a finite polyhedron, and $S_{0}$ a subpolyhedron of $S$. For any subset $P \subset M$ we let $C\left(S, S_{0} ; M, P\right)=C_{P}(M)$ denote the totality of continuous maps $\left(S, S_{0}\right) \rightarrow(M, P)$ with the topology of uniform convergence. Suppose that $P$ is a closed submanifold of $M$ of $\operatorname{codim}(M, P)$ $=p \geqq 1$, and that $(M, P)$ is an oriented pair. Taking for $S_{0}$ a vertex of $S$ we find that $\left(C_{M}(M), C_{P}(M)\right)$ is an oriented pair of codimension $p$, and from the theorem in (A) we have the canonical isomorphism

$$
\phi: H^{i}\left(C_{P}(M)\right) \rightarrow H^{i+p}\left(C_{M}(M), C_{M-P}(M)\right),
$$

with its special multiplicative structure.

Example. We specialize $S$ to be the closed unit interval, and fix a point $m_{0} \in M$. Let $C_{0, P}(M)=\left\{x \in C_{M}(M): x(0)=m_{0}, x(1) \in P\right\}$. Taking into account the contractibility of $C_{0, M}(M)$ we obtain the linking isomorphism

$$
\lambda: H^{i-1}\left(C_{0, M-P}(M)\right) \rightarrow H^{i-p}\left(C_{0, P}(M)\right)
$$

for $i>0$, where $p=\operatorname{codim}(M, P)$. (Let us observe in passing that if $M$ is a Hilbert space $E$, then $C_{0, P}(E)$ (resp., $C_{0, E-P}(E)$ ) has the homotopy type of $P$ (resp., $E-P$ ), and our isomorphism becomes the Alexander duality isomorphism $\lambda: H^{i-1}(E-P) \rightarrow H^{i-p}(P)$ for $i>0$.)

Especially interesting is the situation in which $P, Q$ are closed submanifolds of $M$ with $P$ (resp., $Q$ ) contained in $M-Q$ (resp.,in $M-P$ ) as a deformation retract. Applying the preceding isomorphism twice shows that the cohomology of $C_{0, P}(M)$ is periodic. For instance, let $M$ be the $n$-sphere $S^{n}$ with $P, Q$ antipodal points; then $C_{0, P}\left(S^{n}\right)$ has the homotopy type of the loop space $\Omega S^{n}$, and the linking isomorphism $\lambda: H^{i-1}\left(\Omega S^{n}\right) \rightarrow H^{i-n}\left(\Omega S^{n}\right)$ expresses M. Morse's theorem that $H^{j}\left(\Omega S^{n}\right)=\boldsymbol{Z}$ if $j \equiv 0 \bmod (n-1)$, and 0 otherwise.

Other applications to the computation of the cohomology of path spaces, of spaces of circles, of multiplicative structures (both in cohomology and homology), can be found in [32], [87]. 


\section{BIBLIOGRAPHY}

1. R. Abraham, Transversality in manifolds of mappings, Bull. Amer. Math. Soc. 69 (1963), 470-474.

2. - Lectures of Smale on differential topology, Notes at Columbia University, New York, 1962-1963.

3. V. I. Anosov, Critical points of periodic functionals, Soviet Math. Dokl. 1 (1960), 208-210.

4. R. F. Arens and J. Eells, Jr., On embedding uniform and topological spaces, Pacific J. Math. 6 (1956), 397-403.

5. R. G. Bartle, On the openness and inversion of differentiable mappings, Ann. Acad. Sci. Fenn. 257 (1958), 3-8.

6. A. Bastiani, Applications différentiables et variêtés différentiables de dimension infinie, J. Analyse Math. 13 (1964), 1-114.

7. I. Berstein, J. Eells, Jr. and K. Gęba, Poincaré duality in function spaces, (In preparation).

8. C. Bessaga, Every infinite-dimensional Hilbert space is diffeomorphic with its unit sphere, Bull. Acad. Polon. Sci., (to appear).

9. G. Birkhoff, Analytical groups, Trans. Amer. Math. Soc. 43 (1938), 61-101.

10. R. Bonic and J. Frampton, Differentiable functions on certain Banach spaces, Bull. Amer. Math. Soc. 71 (1965), 393-395.

11. - Smooth functions on Banach manifolds, (to appear).

12. R. Bott, Lectures on $K$-theory, Mimeographed notes, Harvard University, Cambridge, Mass., 1962.

13. N. Bourbaki, Espaces vectoriels topologiques, Hermann, Paris, 1953; Chapters I-II. Hermann, Paris, 1955; Chapters III-IV.

14. H. J. Bremermann, Holomorphic functionals and complex convexity in Banach spaces, Pacific J. Math. 7 (1957), 811-831.

15. F. E. Browder, On a generalization of the Schauder fixed point theorem, Duke Math. J. 26 (1959), 291-304.

16. - Infinite dimensional manifolds and nonlinear elliptic eigenvalue problems, Ann. of Math. 82 (1965), 459-477.

17. - Fixed point theorems on infinite dimensional manifolds, Trans. Amer. Math. Soc. 119 (1965), 179-194.

18. I. Colojoară, On Whitney's imbedding theorem, Rev. Roumaine Math. Pures Appl. 10 (1965), 291-296.

19. P. E. Conner and E. E. Floyd, Differentiable periodic maps, Ergebnisse der Mathematick, Vol. 33, Springer, Berlin, 1964.

20. H. H. Corson, Collections of convex sets which cover a Banach space, Fund. Math. 49 (1961), 143-145.

21. J. Cronin, Fixed points and topological degree in nonlinear analysis, Math. Surveys No. 11, Amer. Math. Soc., Providence, R. I., 1964.

22. J. A. Dieudonné, Recent developments in the theory of locally convex vector spaces, Bull. Amer. Math. Soc. 59 (1953), 495-512.

23. —, Foundations of modern analysis, Academic Press, New York, 1960.

24. J. Dixmier and A. Douady, Champs continus d'espaces hilbertiens et de $C^{*}$ algèbres, Bull. Soc. Math. France 91 (1963), 227-284.

24a. A. Douady, Le problème des modules pour les sous-espaces analytiques compacts d'un espace analytique donné, Sem. Collège de France, 1964-1965. 

367.

25. J. Dugundji, An extension of Tietze's theorem, Pacific J. Math. 1 (1951), 353-

26. N. Dunford and J. T. Schwartz, Linear operators, Interscience, New York, 1958.

27. E. Dynkin, Normed Lie algebras and analytic groups, Uspehi Mat. Nauk. 5 (1950), no. 1 (35) 135-186; Amer. Math. Soc. Transl. Vol. 97 (1953), 66 pp.

28. C. J. Earle and J. Eells, Jr., Foliations and fibrations. On the differential geometry of Teichmüller spaces, (to appear).

29. J. Eells, Jr., On the geometry of function spaces, Symp. Inter. de Topologia Alg., Mexico, 1956; 1958, 303-308.

30. - On submanifolds of certain function spaces, Proc. Nat. Acad. Sci. U.S.A. 45 (1959), 1520-1522.

31. - A class of smooth bundles over a manifold, Pacific J. Math. 10 (1960), 525-538.

32. - Alexander-Pontrjagin duality in function spaces, Proc. Sympos. Pure Math. Vol. III, Amer. Math. Soc., Providence, R. I., 1961, 109-129.

33. - Analysis on manifolds, Mimeographed notes, Cornell University, Ithaca, N. Y., 1964-1965.

34. J. Eells, Jr. and J. H. Sampson, Harmonic mappings of Riemannian manifolds, Amer. J. Math. 86 (1964), 109-160.

35. - - Energie et déformations en géométrie différentielle, Ann. Inst. Fourier (Grenoble) 14 (1964), 61-69.

36. —_ Variational theory in fibre bundles, Proc. Kyoto Conference on Differential Geometry, Kyoto, 1965.

37. J. Frampton, Smooth partitions of unity on Banach manifolds, Ph.D. Thesis, Yale University, New Haven, Conn., 1965.

38. K. Gęba, Sur les groupes de cohomotopie dans les espaces de Banach, C. R. Acad. Sci. Paris 254 (1962), 3293-3295.

39. - Algebraic topology methods in the theory of compact fields in Banach spaces, Fund. Math. 54 (1964), 177-209.

39a. K. Gęba and A. Granas, Algebraic topology in linear normed spaces, Bull. Acad. Polon. Sci. Sér. Sci. Math. Astronom. Phys. I, 13 (1965), 287-290; II, 13 (1965), 341-346.

40. G. Glaeser, Étude de quelques algèbres tayloriennes, J. Analyse Math. 6 (1958), $1-125$.

41. A. Granas, The theory of compact vector fields and some of its applications to topology of functional spaces. I, Rozprawy Mat. 30 (1962).

42. L. Graves, Riemann integration and Taylor's theorem in general analysis, Trans. Amer. Math. Soc. 29 (1927), 163-177.

43. - Implicit functions and differential equations in general analysis, Trans. Amer. Math. Soc. 29 (1927), 514-552.

44. - Topics in functional calculus, Bull. Amer. Math. Soc. 41 (1935), 641-662; 42 (1936), 381-382.

45. L. Graves and T. Hildebrandt, Implicit functions and their differentials in general analysis, Trans. Amer. Math. Soc. 29 (1927), 127-153.

46. N. Grossman, Geodesics on Hilbert manifolds, Ph.D. Thesis, University of Minnesota, Minneapolis, Minn., 1964.

47. - Hilbert manifolds without epiconjugate points, Mimeographed notes, Institute for Advanced Study, Princeton, N. J. 
48. M. Hestenes, Applications of the theory of quadratic forms in Hilbert space to the calculus of variations, Pacific J. Math. 1 (1951), 525-581.

49. - Quadratic variational theory and linear elliptic partial differential equations, Trans. Amer. Math. Soc. 101 (1961), 306-350.

50. E. Hille and R. S. Phillips, Functional analysis and semigroups, Amer. Math. Soc. Colloq. Publ., Vol 31, Amer. Math. Soc., Providence, R. I., 1957.

51. L. Hörmander, Linear partial differential operators, Springer, Berlin, 1963.

52. K. Jänich, Vektorraumbündel und der Raum der Fredholm-Operatoren, Math. Ann. 161 (1965), 129-142.

53. S. Kakutani, Topological properties of the unit sphere in Hilbert space, Proc. Imp. Acad. Tokyo 19 (1943), 269-271.

54. H. H. Keller, Über die Differentialgleichung erster Ordnung in normierten linearen Räurmen, Rend. Circ. Mat. Palermo 8 (1959), 117-144.

55. M. Kerner, Abstract differential geometry, Compositio Math. 4(1937), 308-341.

56. V. L. Klee, Jr., Convex bodies and periodic homeomorphisms in Hilbert space, Trans. Amer. Math. Soc. 74 (1953), 10-43.

57. - Some topological properties of convex sets, Trans. Amer. Math. Soc. 78 (1955), 30-45.

58. W. Klingenberg, The theorem of the three closed geodesics, Bull. Amer. Math. Soc. 71 (1965), 601-605.

59. M. Klingmann, Doctoral Dissertation, University of Heidelberg, 1965.

60. M. A. Krasnosel'skir, Topological methods in the theory of non-linear integral equations, Macmillan, New York, 1964. Translated from the 1956 Russian edition.

61. N. H. Kuiper, The homotopy type of the unitary group of Hilbert space, Topology 3 (1965), 19-30.

62. I. Kupka, Counterexample to the Morse-Sard theorem in the case of infinite dimensional manifolds, Proc. Amer. Math. Soc. 16 (1965), 954-957.

63. J. Kurzweil, An approximation in real Banach spaces, Studia Math. 14 (1954), 214-231.

64. S. Lang, Introduction to differentiable manifolds, Interscience, New York, 1962.

65. D. Laugwitz, Differentialgeometrie ohne Dimensionsaxiom. I, II, Math. Z. 61 (1954), 100-118, 134-149.

66. - Über unendliche kontinuierliche Gruppen. I, Math. Ann. 130 (19551956), 337-350; II, Bayer, Akad. Wiss. Math.-Nat. Kl. 1956, 261-286.

67. —- Grundlagen für die Geometrie der unendlichdimensionalen Finslerräume, Ann. Math. Pura Appl. (4) 41 (1956), 21-41.

68. J. Lelong, Sur les groupes à un paramètre de transformations des variétés différentiables, J. Math. Pures Appl. 37 (1958), 269-278.

69. J. Leray, Sur les équations et les transformations, J. Math. Pures Appl. 24 (1945), 201-248.

70. - La théorie des points fixes et ses applications en analyse, Proc. Internat. Congress, Cambridge, Mass., 1950, 202-208. Vol. 2, Amer. Math. Soc. Providence, R. I., 1952.

71. - Thêorie des points fixes: Indice total et nombre de Lefschetz, Bull. Soc. Math. France 87 (1959), 221-233.

72. J. Leray and J. Schauder, Topologie et équations fonctionnelles, Ann. École Norm. Sup. 51 (1934), 45-78.

73. P. Lévy, Leçons d'analyse fonctionnelle, Gauthiers-Villars, Paris, 1922.

74. B. Maissen, Lie-Gruppen mit Banachräumen als Parameterräume, Acta Math. 108 (1962), 229-269. 
75. J. H. McAlpin, Infinite dimensional manifolds and Morse theory, Ph.D. Thesis, Columbia University, New York, 1965.

76. W. Meyer, Kritische Mannigfaltigkeiten in Hilbert-mannigfaltigkeiten, Doctoral Dissertation, University of Bonn, 1964.

77. A. D. Michal, General differential geometries and related topics, Bull. Amer. Math. Soc. 45 (1939), 525-563.

78. J. W. Milnor, On spaces having the homotopy type of a CW-complex, Trans. Amer. Math. Soc. 90 (1959), 272-280.

79. C. B. Morrey, Jr., Multiple integrals in the calculus of variations, Amer. Math. Soc. Colloq. Lectures, Amherst, Mass., 1964.

80. C. B. Morrey, Jr. and J. Eells, Jr., A variational method in the theory of harmonic integrals. I, Ann. of Math. 63 (1956), 91-128.

81. M. Morse, Abstract variational theory, Memor. Sci. Math. 92 (1939)

82. - Recent advances in variational theory in the large, Proc. Internat. Congress Math., Cambridge, Mass., 1950, 143-156.

83. J. Moser, A new technique for the construction of solutions of nonlinear differential equations, Proc. Nat. Acad. Sci. U.S.A. 47 (1961), 1824-1831.

84. F. J. Murray, On complementary manifolds and projections in spaces $L_{p}$ and $l_{p}$, Trans. Amer. Math. Soc. 41 (1937), 138-152.

85. S. B. Myers, Algebras of differentiable functions, Proc. Amer. Math. Soc. 5 (1954), 917-922.

86. M. Nagumo, Degree of mapping in convex linear topological spaces, Amer. J. Math. 73 (1951), 497-511.

87. I. Namioka, A duality in function spaces, Trans. Amer. Math. Soc. 115 (1965), 131-144.

88. G. Neubauer, Über den Index abgeschlossener Operatoren in Banachräumen, Math. Ann. 160 (1965), 93-130; II, Math. Ann. 162 (1965), 92-119.

89. R. S. Palais, Morse theory on Hilbert manifolds, Topology 2 (1963), 299-340.

90. - On the homotopy type of certain groups of operators, Topology 3 (1965), 271-279.

91. - Lectures on the differential topology of infinite dimensional manifolds, Mimeographed notes, by S. Greenfield, Brandeis University, Waltham, Mass.; 1964-1965.

92. - Homotopy theory of infinite dimensional manifolds, Mimeographed notes, Brandeis University, Waltham, Mass.; Topology 5 (1966), 1-16.

93. —_ Lusternik-Schnirelman theory on Ganach manifolds, Mimeographed notes, Brandeis University, Waltham, Mass.; Topology 5 (1966), 115-132.

94. R. S. Palais and S. Smale, A generalized Morse theory, Bull. Amer. Math. Soc. 70 (1964), 165-172.

95. L. E. Pursell and M. E. Shanks, The Lie algebra of a smooth manifold, Proc. Amer. Math. Soc. 5 (1954), 468-472.

96. C. R. Putnam and A. Wintner, The orthogonal group in Hilbert space, Amer. J. Math. 74 (1952), 52-78.

97. G. Restrepo, Differentiable norms in Banach spaces, Bull. Amer. Math. Soc. 70 (1964), 413-414.

98. E. H. Rothe, Zur Theorie der topologichen Ordnung und der Vektorfelder in Banachschen Räumen, Compositio Math. 5 (1937-1938), 177-197.

99. - Critical points and gradient fields of scalars in Hilbert space, Acta Math. 85 (1951), 73-98. 
100. - Leray-Schauder index and Morse type numbers in Hilbert space, Ann. of Math. 55 (1952), 433-467; 58 (1953), 593-594.

101. - Gradient mappings, Bull. Amer. Math. Soc. 59 (1953), 5-19.

102. - Critical point theory in Hilbert space under general boundary conditions. J. Math. Anal., Appl. 11 (1965), 357-409.

103. H. L. Royden, Function algebras, Bull. Amer. Math. Soc. 69 (1963), 281-298.

104. A. Sard, Hausdorff measure of critical images on Banach manifolds, Amer. J. Math. 87 (1965), 158-174.

105. J. Schauder, Über den Zusammenhang zwischen der Eindeutigkeit und Lösbarkeit. . . Math. Ann. 106 (1932), 661-721.

106. - Invarianz des Gebietes in Funktionalräumen, Studia Math. 1 (1929), 123-139.

107. J. T. Schwartz, Generalizing the Lusternik-Schnirelman theory of critical points, Comm. Pure Appl. Math. 17 (1964), 307-315.

108. - Nonlinear functional analysis, Mimeographed notes, New York University, New York, 1963-1964.

109. I. Segal, Differential operators in the manifold of solutions of a non-linear differential equation, J. Math Pures Appl. 44 (1965), 71-132.

110. S. Smale, Morse theory and a non-linear generalization of the Dirichlet problem, Ann. of Math. 80 (1964), 382-396.

111. - An infinite dimensional version of Sard's theorem. Amer. J. Math. 87 (1965), 861-866.

112. S. L. Sobolev, Applications of functional analysis in mathematical physics, (English transl.) Transl. Math. Monographs, Vol. 7, Amer. Math. Soc. Providence, R. I., 1964.

113. E. H. Spanier, Duality and S-theory, Bull. Amer. Math. Soc. 62 (1956), 194203.

114. N. E. Steenrod, The topology of fibre bundles, Princeton Univ. Press, Princeton, N. J., 1951.

115. A. S. Svarc, The homotopic topology of Banach spaces, Dokl. Akad. Nauk SSSR 154 (1964), 61-63= Soviet Math. Dokl. 5 (1964), 57-59.

116. J. Tomiyama and M. Takesaki, Applications of fibre bundles to the certain class of $C^{*}$-algebras, Tôhoku Math. J. 13 (1961), 498-522.

117. M. M. Vainberg, Variational methods for the study of nonlinear operators, Holden-Day, San Francisco, Calif. 1964. Translated from the 1956 Russian edition.

118. W. T. Van Est and T. J. Korthagen, Non-enlargible Lie algebras, Nederl. Akad. Wetensch. Proc. Ser. A. 26 (1964), 15-31.

119. L. Waelbroek, Le calcul symbolique dans les algèbres commutatives, J. Math. Pures Appl. 33 (1954), 147-186.

120. A. Wasserman, Morse theory for G-manifolds, Bull. Amer. Math. Soc. 71 (1965), 384-388.

121. R. Wood, Banach algebras and Bott periodicity, Topology 4 (1965), 371-389.

Cornell University 